\title{
The invariant charges of the Nambu-Goto String and Canonical Quantization
}

\author{
Dorothea Bahns* \\ Fakultät für Mathematik und Physik \\ der Universität Freiburg \\ Physikalisches Institut \\ Hermann-Herder-Straße 3 \\ D-79104 Freiburg, Germany
}

\begin{abstract}
It is shown that the algebra of diffeomorphism-invariant charges of the Nambu-Goto string cannot be quantized in the framework of canonical quantization. The argument is shown to be independent of the dimension of the underlying Minkowski space.
\end{abstract}

\section{Introduction}

The action of the Nambu-Goto string is a generalization of the reparametrization-invariant action of the relativistic particle in $d$-dimensional Minkowski space, where instead of a point-particle, a one-dimensional extended object (a string) is considered. Correspondingly, the solutions of the equations of motion are surfaces swept out by the string in spacetime (called world-sheets) which are extremal with respect to the Minkowski metric. The parametrization of these surfaces is not fixed by the equations of motion, and hence, a change of the parametrization corresponds to a symmetry transformation which does not change the physical state of the system. Therefore, the Nambu-Goto string is a system with gauge group given by the diffeomorphisms of a surface. As such, it provides an interesting model to study the fundamental problem of quantizing a system with gauge freedom given by the diffeomorphism group.

For closed strings, the world-sheet is tube-shaped. It was shown especially in this case, that the Nambu-Goto string can be treated as an integrable system and that its integrals of motion can be constructed from a suitably defined monodromy [2]. These integrals of motion are functionals on the world-sheet which are invariant under arbitrary reparametrizations (gauge transformations) and as such are observable quantities. They form a graded Poisson algebra [3, 4], the Poisson algebra of invariant charges, and were shown to be complete in the sense that, up to translations in the direction of its total energy-momentum vector, the string can be reconstructed from the knowledge of the invariant charges, together with the infinitesimal generators of boosts [5]. In this scheme, the constraints which are present in the system enter as a condition on the representation of the algebra, and - together with conditions regarding Hermiticity and positivity of the energy - distinguish its physically meaningful representations.

*bahns@mail.desy.de 
The algebra of invariant charges provides the starting point of the algebraic quantization of the Nambu-Goto string [2]. This scheme is based on the idea that the correspondence principle should be applied to physically meaningful quantities only, which in a theory with gauge freedom means that it is applicable only to gauge-invariant observables. In this spirit, the graded Poisson algebra of invariant charges of the Nambu-Goto string is quantized by application of the correspondence principle, replacing the Poisson brackets by commutators and allowing for particular (observable) quantum corrections which are restricted by demanding structural similarity of the classical and the quantum algebra. So far, it does not seem at all likely that in this scheme an obstruction regarding the dimension $d$ of the underlying Minkowski space should appear (other than $d>2$ ). In contrast to this, the canonical quantization of the Nambu-Goto string is consistent only in certain critical dimensions. Here, the correspondence principle is assumed to hold for the Fourier modes of some particular parametrization, i.e. for quantities which are not observable. It leads to the well-known construction of Fock space which contains the physically relevant states as a subspace.

In this paper, which is an exposition of results gained some years ago [1, it is shown that canonical quantization does not yield a representation of the algebra of invariant charges. After a short exposition of known results regarding the algebraic approach to the quantization of the Nambu-Goto string [3, 4, 6] in the the following two sections, the fourth section contains an investigation of the canonical quantization and its application to the algebra of invariant charges. It is shown that unobservable anomalies arise in the defining relations of the algebra in $3+1$ dimensions. In section 5 it is then shown that the problem cannot be cured by adjusting the dimension of the underlying Minkowski space.

\section{The Poisson algebra of invariants}

In the Hamiltonian formalism, the fact that the world-sheet is independent of the particular parametrization chosen to describe it, becomes manifest in the appearance of two primary constraints which are the infinitesimal generators of gauge transformations (reparametrizations). The canonical momenta $p_{\mu}$ and positions $x_{\mu}, \mu=0, \ldots, d-1$ are not independent of each other, and the canonical Hamilton function vanishes. Following Dirac's treatment of systems with constraints, a total Hamiltonian $H_{T}$ is introduced which is a linear combination of the two primary constraints with two Lagrangian multipliers $\alpha$ and $\beta$. Here, we specialize to the case where $\alpha$ and $\beta$ do not depend on the original degrees of freedom $x_{\mu}$ and $p_{\mu}$. The dynamics of the string is thus governed by the gauge freedom only, and fixing the two Lagrangian multipliers corresponds to fixing a gauge. It follows that integrals of motion of $H_{T}$ are gauge-invariant quantities, i.e. invariant charges which do not depend on the parametrization. In [2] it was shown that by treating the string as an integrable system, such invariant charges arise as (symmetric polynomials of) the eigenvalues of a monodromy matrix of a system of linear differential equations whose compatibility condition (a "zero curvature condition") is equivalent to the equations of motion of the string.

It is convenient to express the equations of motion as well as the constraints in terms of left and right movers $u_{\mu}^{ \pm}(\tau, \sigma)=p_{\mu}(\tau, \sigma) \pm \frac{1}{2 \pi \alpha^{\prime}} \partial_{\sigma} x_{\mu}(\tau, \sigma), \mu=0, \ldots, d-1$, where $1 / 2 \pi \alpha^{\prime}$ is the string tension. Here, a foliation is chosen such that $\partial_{\tau} x_{\mu}(\tau, \sigma)$ is a timelike vector and $\partial_{\sigma} x_{\mu}(\tau, \sigma)$ is spacelike, $\sigma \in[0, \omega(\tau))$, where $\omega(\tau)$ is the period of the string's parametrization 
(as a function of $\sigma$ at fixed $\tau$ ). The constraints are then equivalent to demanding that $u^{ \pm}$be lightlike. With left and right movers, an invariant charge is given by the following explicit expression,

$$
\begin{aligned}
\mathcal{Z}_{\mu_{1} \cdots \mu_{N}}^{ \pm}(\tau, \sigma) & \stackrel{\text { def }}{=} \mathcal{R}_{\mu_{1} \cdots \mu_{N}}^{ \pm}(\tau, \sigma)+\mathcal{R}_{\mu_{2} \cdots \mu_{N} \mu_{1}}^{ \pm}(\tau, \sigma)+\cdots+\mathcal{R}_{\mu_{N} \mu_{1} \cdots \mu_{N-1}}^{ \pm}(\tau, \sigma) \\
& =\int_{\sigma}^{\sigma+\omega(\tau)} d \sigma^{\prime} u_{\mu_{1}}^{ \pm}\left(\tau, \sigma^{\prime}\right) \mathcal{R}_{\mu_{2} \cdots \mu_{N}}^{ \pm}\left(\tau, \sigma^{\prime}\right)
\end{aligned}
$$

where

$$
\mathcal{R}_{\mu_{1} \cdots \mu_{N}}^{ \pm}(\tau, \sigma)=\int_{\sigma}^{\sigma+\omega(\tau)} d \sigma_{1} u_{\mu_{1}}^{ \pm}\left(\tau, \sigma_{1}\right) \int_{\sigma}^{\sigma_{1}} d \sigma_{2} u_{\mu_{2}}^{ \pm}\left(\tau, \sigma_{2}\right) \cdots \int_{\sigma}^{\sigma_{N-1}} d \sigma_{N} u_{\mu_{N}}^{ \pm}\left(\tau, \sigma_{N}\right)
$$

From the equations of motion of the monodromy matrices (see [4]), one finds

$$
\begin{aligned}
\partial_{\sigma} \mathcal{R}_{\mu_{1} \cdots \mu_{N}}^{ \pm}(\tau, \sigma) & =u_{\mu_{1}}^{ \pm}(\tau, \sigma) \mathcal{R}_{\mu_{2} \cdots \mu_{N}}^{ \pm}(\tau, \sigma)-\mathcal{R}_{\mu_{1} \cdots \mu_{N-1}}^{ \pm}(\tau, \sigma) u_{\mu_{N}}^{ \pm}(\tau, \sigma) \\
\partial_{\tau} \mathcal{R}_{\mu_{1} \cdots \mu_{N}}^{ \pm}(\tau, \sigma) & =(\alpha \pm \beta)_{(\tau, \sigma)} \partial_{\sigma} \mathcal{R}_{\mu_{1} \cdots \mu_{N}}^{ \pm}(\tau, \sigma)
\end{aligned}
$$

such that indeed,

$$
\partial_{\sigma} \mathcal{Z}_{\mu_{1} \cdots \mu_{N}}^{ \pm}(\tau, \sigma)=\partial_{\tau} \mathcal{Z}_{\mu_{1} \cdots \mu_{N}}^{ \pm}(\tau, \sigma)=0 .
$$

Another way to express the fact that the functionals $\mathcal{Z}$ are gauge invariant is that they Poisson-commute with the total Hamiltonian, $\left\{\mathcal{Z}_{\mu_{1} \cdots \mu_{N}}^{ \pm}, H_{T}\right\}_{0}=0$. Here, the Poisson bracket is derived from the canonical Poisson bracket $\left\{x_{\mu}, p_{\nu}\right\}_{0}$, such that (for fixed $\tau$, where w.l.o.g. $\omega(\tau)=2 \pi$ and with the periodic $\delta$-distribution $\delta_{2 \pi}$ ),

$$
\left\{u_{\mu}^{ \pm}(\tau, \sigma), u_{\nu}^{ \pm}\left(\tau, \sigma^{\prime}\right)\right\}_{0}= \pm \frac{1}{2 \pi \alpha^{\prime}} 2 \eta_{\mu \nu} \partial_{\sigma} \delta_{2 \pi}\left(\sigma-\sigma^{\prime}\right), \quad \text { all others } 0 .
$$

The invariant charges were shown to form a Poisson algebra with respect to this bracket 3 , 4. From the knowledge of the invariants, together with the generators of boosts, the string can be reconstructed up to translations in the direction of its total energy-momentum vector $^{1}$ and in this sense, the invariant charges are complete [5].

Let us now turn to an exposition of the structure of the Poisson algebra of invariant charges [6]. An invariant charge $\mathcal{Z}_{\mu_{1} \ldots \mu_{N}}$ can be split into a sum of so-called homogeneous invariants $\mathcal{Z}_{\mu_{1} \ldots \mu_{N}}^{(K)}$ of order $K=1, \ldots, N$, which arise from powers of the logarithm of the monodromy matrices [3, 4] and are themselves invariant under arbitrary reparametrizations,

$$
\mathcal{Z}_{\mu_{1} \cdots \mu_{N}}^{ \pm}=\sum_{K=1}^{N} \mathcal{Z}_{\mu_{1} \cdots \mu_{N}}^{ \pm(K)}
$$

The only invariant charge of order $K=1$ is the total momentum, $\mathcal{Z}_{\mu}^{-(1)}=\mathcal{Z}_{\mu}^{+(1)}=$ $\oint d \sigma_{1} u_{\mu}^{ \pm}\left(\tau, \sigma_{1}\right)=\mathcal{P}_{\mu}$. It is the only invariant charge which is an element of both the algebra built from left movers and the one built from right movers, and it Poisson-commutes with all (homogeneous) invariant charges. In what follows, only massive strings will be

\footnotetext{
${ }^{1}$ This non-uniqueness is due to the fact that the construction of the $\mathcal{Z}$ relies only on $\partial_{\sigma} x$, not on $x$ itself. If the string splits into different parts or if two strings collide [7, an absolute position, the splitting or meeting point, enters.
} 
considered, where $\mathcal{P}^{2}=\mathfrak{m}^{2}$, and we pass to the rest frame of the string where $\mathcal{P}_{\mu}=$ $(\mathfrak{m}, 0, \ldots, 0), \mathfrak{m}>0$. By (2.5), the algebra built from left movers and the one built from right movers Poisson-commute with one another, and their structure constants differ only by signs. It is therefore sufficient to analyse the right mover part (referred to as $\mathfrak{h}$ ) only. Analogous results then hold also for the left mover part. The algebra $\mathfrak{h}$ is graded under the action of the Poisson bracket $\{\cdot, \cdot\}$ which, compared to the canonical one, is rescaled by a factor $2 \pi \alpha^{\prime}$,

$$
\mathfrak{h}=\bigoplus_{\ell=0}^{\infty} \mathfrak{V}^{\ell}(\mathfrak{h}), \quad\left\{\mathfrak{V}^{\ell_{1}}, \mathfrak{V}^{\ell_{2}}\right\} \subset \mathfrak{V}^{\ell_{1}+\ell_{2}}, \quad \mathfrak{V}^{\ell_{1}} \cdot \mathfrak{V}^{\ell_{2}} \subset \mathfrak{V}^{\ell_{1}+\ell_{2}+1}, \quad \ell=N-K-1
$$

where each $\mathfrak{V}^{\ell}$ is finite dimensional as a vector space. A parity operator is defined on $\mathfrak{h}$ which assigns positive (negative) parity to an invariant which contains an even (odd) number of spacelike indices. Each $\mathfrak{V}^{\ell}$ splits up into a direct sum of a space with even $\left(\mathfrak{V}_{+}^{\ell}\right)$ or odd $\left(\mathfrak{V}_{-}^{\ell}\right)$ parity (one of which may be trivial). The vector space $\mathfrak{V}^{0}$ is $(d-1)$ dimensional and forms a subalgebra isomorphic to the Lie algebra $s o(d-1)$, the Lie algebra of the stabilizer group of $\mathcal{P}_{\mu}$. All vector spaces $\mathfrak{V}_{ \pm}^{\ell}$ are invariant under the Poisson action of $\mathfrak{V}^{0}$, and therefore, each of them carries a linear representation of $s o(d-1)$ and can be decomposed into a direct sum of isotypical components (corresponding to different spins and parities).

By a well-scrutinized conjecture, which has been proved for $d=3$ up to degree $\ell=7$, any invariant charge can be expressed as a polynomial in certain standard invariants, and the number of standard invariants in each level $\mathfrak{V}^{\ell}$ is known. It was shown, however, in [3, 4] that some invariant charges, the so-called exceptional elements, cannot be expressed in terms of Poisson brackets of standard invariants of lower degrees. Moreover, a major complication in the investigation of $\mathfrak{h}$ is that taking a Poisson bracket of two standard invariants, one in general obtains not only a standard invariant, but moreover a linear combination of products of other standard-invariants, whence the standard invariants do not form a Lie algebra. In fact, it was shown that there is no algebraic basis which would render $\mathfrak{h}$ as the enveloping algebra of a Lie algebra 3, 4]. Instead, it is necessary to generate $\mathfrak{h}$ by (multiple) Poisson brackets as well as by products of a set of generating invariant charges. The generating invariant charges do not freely generate $\mathfrak{h}$, and relations (other than those given by antisymmetry and the Jacobi identity) between (multiple) Poisson brackets and products persist. Their number at given degree $\ell$ is equal to $m_{\ell}-n_{\ell}$, where $n_{\ell}$ is the number of standard invariants in $\mathfrak{V}^{\ell}$ and $m_{\ell}$ the number of Hall-basis elements ${ }^{2}$ in $\mathfrak{V}^{\ell}$ which can be built from standard invariants of lower degree than $\ell$, see [6].

In what follows, $d=4$ spacetime dimensions are considered and some of the structural insight gained in [6] is reproduced. The set of generating invariants in $d=4$ is given by $3+14$ invariants from $\mathfrak{V}^{0}$ and $\mathfrak{V}^{1}$, respectively, which generate a subalgebra $\mathfrak{U}$ of $\mathfrak{h}$, together with the (modified) exceptional elements $B_{0}^{(\ell)}, \ell=1,3,5, \ldots$, which form an Abelian subalgebra of $\mathfrak{h}$, and act semidirectly on $\mathfrak{U}$. In the present investigation only $\ell \leq 2$ will be considered, where these claims were proved rigorously. Employing an angular momentum (or rather a spin) basis $\left\{e_{0}, e_{ \pm}=\frac{1}{\sqrt{2}}\left(e_{1} \pm i e_{2}\right), e_{3}\right\}$ in $\mathbb{R}^{4}$, we obtain the following generating

\footnotetext{
${ }^{2}$ This means that only such brackets are considered which cannot be transformed into each other by antisymmetry or by the Jacobi identity.
} 
invariants for the vector space basis of $\mathfrak{V}^{0}$ :

$J_{1,1}=\frac{-1}{4 \mathfrak{m}}\left(i \mathcal{Z}_{0+3}^{(2)}-i \mathcal{Z}_{03+}^{(2)}\right), J_{1,0}=\frac{-1}{4 \mathfrak{m}}\left(i \mathcal{Z}_{0+-}^{(2)}-i \mathcal{Z}_{0-+}^{(2)}\right), J_{1,-1}=\frac{-1}{4 \mathfrak{m}}\left(i \mathcal{Z}_{0-3}^{(2)}-i \mathcal{Z}_{03-}^{(2)}\right)$.

As a vector space, $\mathfrak{V}^{1}$ is spanned by

$$
\left(J_{1}^{2}\right)_{0}, B_{0}^{(1)}, S_{1} \text { and }\left(J_{1}^{2}\right)_{2}, T_{2}, S_{2},
$$

which are multiplets of $s o(3)$ with spin $J=0,1$ and 2, respectively, with the 14 generating invariants given by:

$$
\begin{aligned}
& B_{0}^{(1)}=\mathcal{Z}_{0-0+}^{(2)}+\frac{1}{2} \mathcal{Z}_{0303}^{(2)} \\
& T_{2}=\left\{T_{2, m} \mid m=-2, \ldots, 2\right\} \quad \text { with } T_{2,-2}=\frac{1}{2} \mathcal{Z}_{00--}^{(2)} \\
& S_{2}=\left\{S_{2, m} \mid m=-2, \ldots, 2\right\} \quad \text { with } S_{2,-2}=i \mathcal{Z}_{03--}^{(2)} \\
& S_{1}=\left\{S_{1, m} \mid m=-1, \ldots, 1\right\} \quad \text { with } S_{1,-1}=\mathcal{Z}_{0+--}^{(2)}-\mathcal{Z}_{0-33}^{(2)} \\
& \text { with } i\left\{J_{1, \pm 1}, X_{j, m}\right\}=\mp \frac{1}{\sqrt{2}} \sqrt{(j \pm m+1)(j \mp m)} X_{j, m \pm 1}
\end{aligned}
$$

and where

$$
\left(X_{j_{1}} \cdot Y_{j_{2}}\right)_{j, m}=\underbrace{\sum_{m_{1}=-j_{1}}^{j_{1}} \sum_{m_{2}=-j_{2}}^{j_{2}}}_{m_{1}+m_{2}=m}\left\langle j, m \mid j_{1}, m_{1} ; j_{2}, m_{2}\right\rangle X_{j_{1}, m_{1}} \cdot Y_{j_{2}, m_{2}} .
$$

Here, the Clebsch-Gordan coefficients $\left\langle j, m \mid j_{1}, m_{1} ; j_{2}, m_{2}\right\rangle$ are defined with conventions of Condon and Shortley. The action of $J_{1,1}$ respects the parity, the tensor rank $N$ as well as the order $K$, and hence, the basis elements with higher magnetic numbers than $m=-J$ are indeed again invariants of the same parity, tensor rank $N=4$ and order $K=2$, whose explicit form can be calculated using (2.5). Complex conjugation yields an involution on the algebra, and the phases of the generating invariants are chosen such that for $m=0$ they are real, $X_{j, m}^{*}=(-1)^{m} X_{j,-m}$.

The vector space basis of $\mathfrak{V}^{2}$ is again given by products and Poisson brackets of the above generating invariant charges. By (2.6), such Poisson brackets can only be single brackets built from elements of $\mathfrak{V}^{1}$. The number of standard invariants in $\mathfrak{V}^{1}$ being 14, $\frac{1}{2} \cdot 14 \cdot 13=91$ such brackets can be formed, if the antisymmetry of the bracket is taken into account (no dependences from the Jacobi identity arise, since no multiple brackets appear). The number of standard invariants in $\mathfrak{V}^{2}$ being 40, 51 algebraic relations between these brackets persist, which by the above remarks will also involve products of generators. They were given in [6] and are reproduced in appendix A. The relations, which are real, are organized in 9 multiplets, and it follows that only 9 relations are truly independent, while the others can be produced by the action of $\mathfrak{V}^{0}$.

\section{Algebraic quantization by correspondence}

The basic idea of the algebraic approach is that the correspondence principle is physically meaningful only for observable (i.e. gauge-invariant) quantities. Since it provides an 
alternative to the canonical quantization scheme of the Nambu-Goto string, the general idea is reproduced here, cf. [6. The classical Poisson algebra with commutative multiplication is to be deformed into an associative algebra, where Poisson brackets are replaced by commutators and certain quantum corrections are admitted, which are restricted by demanding structural similarity of the classical and the quantum algebra (see below). In particular, it is required that the number of independent relations should not be changed. In principle, this quantization scheme is applicable in arbitrary dimensions, but the calculations used in this paper have been performed in $1+3$ dimensions. In a first step, it is assumed that (dimensionless) quantum generators $\hat{X}_{1}, \hat{\mathbb{X}}_{2}, \hat{S}_{2}, \hat{S}_{1}, \hat{\mathbb{B}}_{0}(\ell), \ell=1,3, \ldots$, exist which correspond to the classical ones (when scaled by factors $\left(\hbar / 2 \pi \alpha^{\prime}\right)^{\ell+1}$ ). The quantum version of a classical relation at order $\ell$ is then obtained as follows:

- Replace each rescaled Poisson bracket by a commutator $[\cdot, \cdot]$ (multiplied with a factor $\left.2 \pi \alpha^{\prime} / i \hbar\right)$ without changing the order of the bracket's entries. The action of $\mathfrak{V}^{0}$ on higher levels $\mathfrak{V}^{\ell}$ remaining the same, this replacement can be done for the full multiplet. Replace the multiplication by anticommutators $\{\cdot, \cdot\}$ (multiplied with a factor $\left.\frac{1}{2}\right)$.

- By construction, the resulting relation consists of (anti-)commutators of the dimensionless generators, multiplied by a global factor $\left(\hbar / 2 \pi \alpha^{\prime}\right)^{(\ell+1)}$. Now quantum corrections are admitted which have the same spin and parity as the relation under consideration but are of lower degree. They enter the relation multiplied by an appropriate positive power of $\hbar$ as well as with parameters which respect the reality property of the relation and are restricted by the structural similarity conditions (see below).

As an example, we consider the classical relation with $J^{P}=1^{-}$involving $B_{0}^{(1)}$,

$$
\left\{B_{0}^{(1)}, S_{1}\right\}_{1}=-i 6 \sqrt{\frac{2}{5}}\left\{T_{2}, S_{2}\right\}_{1}+2 \sqrt{\frac{3}{5}}\left\{T_{2}, S_{1}\right\}_{1}-24 \sqrt{\frac{3}{5}}\left(J_{1} \cdot S_{2}\right)_{1}+i 12 \sqrt{2}\left(J_{1} \cdot S_{1}\right)_{1},
$$

where Poisson brackets and products are multiplets of spin $J=1$ as given in formulas (A.21) and (A.22) in appendix A This relation is replaced by

$$
\begin{aligned}
{\left[\hat{\mathbb{R}}_{0}{ }^{(1)}, \hat{\mathbb{S}}_{1}\right]_{1}=} & -i 6 \sqrt{\frac{2}{5}}\left[\hat{\mathbb{X}}_{2}, \hat{\mathbb{S}}_{2}\right]_{1}+2 \sqrt{\frac{3}{5}}\left[\hat{\mathbb{K}}_{2}, \hat{\mathbb{S}}_{1}\right]_{1}-i 12 \sqrt{\frac{3}{5}}\left\{\hat{\mathbb{X}}_{1}, \hat{\mathbb{S}}_{2}\right\}_{1} \\
& -6 \sqrt{2}\left\{\hat{\mathbb{X}}_{1}, \hat{\mathbb{S}}_{1}\right\}_{1}+i d \hat{\mathbb{S}}_{1}
\end{aligned}
$$

with a real (in fact, rational) parameter $d$ and multiplets of (anti-)commutators with spin $J=1$. Note that in quantum relations, $\{\cdot, \cdot\}$ denotes the anticommutator, not the Poisson bracket. Let us now consider the requirement of structural similarity of the classical and the quantized algebra which puts restrictions on these parameters. In order to compare two relations, they have to be brought into some standard form, and the multiplication now being noncommutative, it is clear that in doing so, one may pick up correction terms of lower order, for instance, (see [6]):

$$
\begin{aligned}
& \left\{\left\{A_{j_{1}}, B_{j_{2}}\right\}_{j}, C_{j_{3}}\right\}_{J}=\sum_{k}(-)^{k+j_{2}+j_{3}} \sqrt{(2 j+1)(2 k+1)} \\
& \quad\left((-)^{j+1}\left\{\begin{array}{ccc}
j_{2} & j_{1} & j \\
j_{3} & J & k
\end{array}\right\}\left[\left[\mathbf{A}_{\mathbf{j}_{1}}, \mathbf{C}_{\mathbf{j}_{3}}\right]_{\mathbf{k}}, \mathbf{B}_{\mathbf{j}_{2}}\right]_{\mathbf{J}}+\left\{\begin{array}{ccc}
j_{1} & j_{2} & j \\
j_{3} & J & k
\end{array}\right\}\left\{\left\{B_{j_{2}}, C_{j_{3}}\right\}_{k}, A_{j_{1}}\right\}_{J}\right)
\end{aligned}
$$


with $6 j$-symbols $\{:::\}$ (the correction term is printed in boldface letters). By this mechanism, new dependences between these lower order correction terms may arise, which do not possess a classical analogue. It has been shown explicitly up to the fifth degree [6, 8, that these dependences can be trivially fulfilled or can be reduced to old dependences by fixing the parameters in the quantum relations in a suitable way. In $\ell=2$, all but one (which appears in the relation with $J^{P}=1^{+}$) have been shown to be trivial. The elements of the quantized algebra are again referred to as observables.

In [9, 10] it was shown that a quantization which is consistent with the relations found so far is possible to all orders $\ell$, provided that certain hypotheses concerning the classical algebra (as sketched in the preceeding section) are true. Here, an a posteriori approach was pursued, namely to use an explicit infinite dimensional embedding Lie algebra whose elements are not necessarily invariant under reparametrizations, but which is distinguished by the fact that, restricted to its reparametrization invariant elements, it provides a concrete realization of the quantum algebra of observables found in [6].

\section{Canonical Quantization}

The canonical quantization of the Nambu-Goto string is by far more popular than the approach described above. Its virtue is that it is much simpler. On the other hand, it has some undesirable features, for instance, to name but two, the appearance of a critical dimension and the impossibility to fully implement the constraints. While the general theory of strings has by now evolved into an elaborate theory in its own right and has moved away from the original Nambu-Goto action, it is still worthwhile to consider the fundamental question of whether, in the presence of the alternative approach of algebraic quantization, the canonical quantization scheme is apt to capture the reparametrization invariance of the Nambu-Goto string. It it the aim of the following sections to show that the Fourier modes of an arbitrary parametrization do not provide a suitable starting point for the quantization of the algebra of invariant charges.

\subsection{Classical Fourier modes}

In order to fix the notation, the well-known decomposition of left and right movers $u_{\mu}^{ \pm}$, $\mu=0, \ldots, d-1$, into Fourier modes is reproduced here,

$$
\begin{aligned}
& u_{\mu}^{-}(\tau, \sigma)=p_{\mu}(\tau, \sigma)-\frac{1}{2 \pi \alpha^{\prime}} \partial_{\sigma} x_{\mu}(\tau, \sigma)=\frac{\mathcal{P}_{\mu}}{2 \pi}+\frac{\mathfrak{m}}{2 \pi} \sum_{n>0}\left(\alpha_{\mu}^{n}(\tau) e^{i n \sigma}+\alpha_{\mu}^{-n}(\tau) e^{-i n \sigma}\right) \\
& u_{\mu}^{+}(\tau, \sigma)=p_{\mu}(\tau, \sigma)+\frac{1}{2 \pi \alpha^{\prime}} \partial_{\sigma} x_{\mu}(\tau, \sigma)=\frac{\mathcal{P}_{\mu}}{2 \pi}+\frac{\mathfrak{m}}{2 \pi} \sum_{n>0}\left(\beta_{\mu}^{n}(\tau) e^{-i n \sigma}+\beta_{\mu}^{-n}(\tau) e^{i n \sigma}\right),
\end{aligned}
$$

with $\left(\alpha_{\mu}^{-n}\right)^{*}=\alpha_{\mu}^{n}$ and $\left(\beta_{\mu}^{-n}\right)^{*}=\beta_{\mu}^{n}$. The zero modes $\alpha_{\mu}^{0}$ and $\beta_{\mu}^{0}$ are equal to $\mathcal{P}_{\mu} / 2 \pi$, since the positions' zero mode is independent of $\sigma$ and hence vanishes in $\partial_{\sigma} x$. Note that in the conformal gauge, where $2 \pi \alpha^{\prime} p(\tau, \sigma)=\partial_{\tau} x(\tau, \sigma)$, the components' dependence on $\tau$ is given as follows:

$$
\alpha_{\mu}^{ \pm n}(\tau)=\alpha_{\mu}^{ \pm n} e^{\mp i n \tau} \quad \text { and } \quad \beta_{\mu}^{ \pm n}(\tau)=\beta_{\mu}^{ \pm n} e^{\mp i n \tau} .
$$

In what follows, the dependence on $\tau$ is suppressed, and we write $\alpha_{\mu}^{ \pm n}$ for $\alpha_{\mu}^{ \pm n}(\tau)$. With conventions as above, we find the following (un-rescaled) Poisson brackets,

$$
\left\{\alpha_{\mu}^{m}, \alpha_{\nu}^{-n}\right\}_{0}=-\frac{4 \pi}{2 \pi \alpha^{\prime} \mathfrak{m}^{2}} i n \eta_{\mu \nu} \delta_{m, n} \quad \text { and } \quad\left\{\beta_{\mu}^{m}, \beta_{\nu}^{-n}\right\}_{0}=-\frac{4 \pi}{2 \pi \alpha^{\prime} \mathfrak{m}^{2}} i n \eta_{\mu \nu} \delta_{m, n}
$$


all others 0 . It is important to bear in mind that, apart from the zero mode $\mathcal{P}_{\mu} / 2 \pi$, the Fourier coefficients depend on the chosen parametrization. Nonetheless, it is of course possible that certain polynomials in the coefficients are independent of the parametrization. Prominent examples are the generators of the Poincaré group. Moreover, as was analysed in [5] and further elaborated in [11, the classical invariant charges $\mathcal{Z}$ can be expressed as polynomials of Fourier modes: Inserting the decomposition (4.8) in (2.1), we find (for the left mover part of the algebra, and likewise for the right mover part),

$$
\begin{aligned}
\mathcal{Z}_{\mu_{1} \ldots \mu_{N}} & =\frac{\mathfrak{m}^{N}}{(2 \pi)^{N}} \sum_{n_{1}=-\infty}^{\infty} \ldots \sum_{n_{N}=-\infty}^{\infty} \alpha_{\mu_{1}}^{n_{1}} \cdots \alpha_{\mu_{N}}^{n_{N}} \oint d \sigma_{1} e^{i n_{1} \sigma_{1}} \int_{\sigma_{1}}^{\sigma_{1}+2 \pi} d \sigma_{2} e^{i n_{2} \sigma_{2}} \ldots \int_{\sigma_{1}}^{\sigma_{N-1}} d \sigma_{N} e^{i n_{N} \sigma_{N}} \\
& =\frac{\mathfrak{m}^{N}}{(2 \pi)^{N}} \sum_{n_{1}=-\infty}^{\infty} \ldots \sum_{n_{N}=-\infty}^{\infty} \alpha_{\mu_{1}}^{n_{1}} \ldots \alpha_{\mu_{N}}^{n_{N}} \sum_{K=1}^{N} \frac{(2 \pi)^{K}}{(K-1) !}\left(\frac{1}{i}\right)^{N-K} C_{n_{1} \ldots n_{N}}^{[K, N]},
\end{aligned}
$$

where by (2.4), the starting point of the last integration is irrelevant, and where without loss of generality, $\omega(\tau)=2 \pi$. Following [11, the iterated integrals are replaced by a sum over combinatorial factors $C_{n_{1} \ldots n_{N}}^{[K, N]}$ with the following properties:

i.) cyclic symmetry in $n_{1}, \ldots, n_{N}$

ii.) recursion relation:

$$
C_{n_{1} \ldots n_{N}}^{[K, N]}=\frac{1}{n_{N}}\left(C_{n_{1} \ldots n_{N-2} n_{N-1}+n_{N}}^{[K, N-1]}-C_{n_{1}+n_{N} n_{2} \ldots n_{N-1}}^{[K, N-1]}\right) \quad \text { for } n_{N} \neq 0
$$

iii.)

$$
C_{n_{1} \ldots n_{N}}^{[N, N]}=\delta_{n_{1}, 0} \cdots \delta_{n_{N}, 0} \quad \text { and } \quad C_{0 \ldots 0}^{[K, N]}=\delta_{K, N}
$$

Due to the recursion relation, the combinatorial coefficients are in general linear combinations of products of Kronecker symbols (with rational coefficients). It follows from the definitions that the Fourier decomposition of homogeneous invariants is given as follows,

$$
\mathcal{Z}_{\mu_{1} \ldots \mu_{N}}^{(K)}=\frac{\mathfrak{m}^{N}}{(2 \pi)^{N-K}} \frac{1}{(K-1) !}\left(\frac{1}{i}\right)^{N-K} \sum_{n_{1}=-\infty}^{\infty} \ldots \sum_{n_{N}=-\infty}^{\infty} \alpha_{\mu_{1}}^{n_{1}} \ldots \alpha_{\mu_{N}}^{n_{N}} C_{n_{1} \ldots n_{N}}^{[K, N]} .
$$

It is important to note that the degree $\ell=N-K-1$ of the homogeneous invariant can be determined only by the inverse power of the factor $2 \pi$ (minus 1 ), while its tensor rank $N$ is encoded in the power of the mass $\mathfrak{m}$. The rest system is implemented by requesting that $\alpha_{\mu}^{n=0}=\delta_{\mu, 0}$.

The reader is assumed to be familiar with the canonical approach and hence it is only mentioned that in this approach, the decomposition of left and right movers in Fourier modes leads to the following decomposition of the constraints,

$$
0 \approx \pi \alpha^{\prime}\left(u^{-}\right)^{2}=: \sum_{n=-\infty}^{\infty} \bar{L}^{n} e^{i n \sigma}, \quad 0 \approx \pi \alpha^{\prime}\left(u^{+}\right)^{2}=: \sum_{n=-\infty}^{\infty} L^{-n} e^{i n \sigma},
$$

where $L^{n}$ and $\bar{L}^{n}$ are generators of (two copies of) the Witt algebra. 


\subsection{Normal ordering of the invariant charges}

In the canonical quantization procedure, the correspondence principle is applied to the (non-observable) Fourier modes. They are replaced by operators on Fock space, with positive modes corresponding to annihilation operators, and negative modes corresponding to creation operators (such that in the conformal gauge, $e^{-i n \tau}, n>0$, belongs to an annihilation operator). Zero modes correspond to multiples of the identity and normal ordering is used to define monomials of operators. The Poisson brackets (4.10) are replaced by commutators $\frac{1}{i \hbar}[\cdot, \cdot]$, such that

$$
\left[\alpha_{\mu}^{m}, \alpha_{\nu}^{-n}\right]=\hbar \frac{4 \pi}{2 \pi \alpha^{\prime} \mathfrak{m}^{2}} n \eta_{\mu \nu} \delta_{m, n} \quad \text { and } \quad\left[\beta_{\mu}^{m}, \beta_{\nu}^{-n}\right]=\hbar \frac{4 \pi}{2 \pi \alpha^{\prime} \mathfrak{m}^{2}} n \eta_{\mu \nu} \delta_{m, n},
$$

all others 0 . The consequences of this quantization procedure for the Witt algebra are well-known. It yields a nontrivial central extension of it, the so-called Virasoro algebra, and due to the appearance of the central charge, it is not possible to define the physical subspace of the Fock space as the kernel of all generators : $L^{n}:$ and $: \bar{L}^{n}:$, but only of those with $n \geq-1$ (alternatively of those with $n \leq 1$ ). For later use, the explicit form of a generator with $n>0$ is reproduced here,

$$
: \bar{L}^{n}:=\frac{\alpha^{\prime}}{2} \mathfrak{m} \mathcal{P} \cdot \alpha^{n}+\frac{\alpha^{\prime}}{4} \mathfrak{m}^{2} \sum_{m=1}^{n-1} \alpha^{m} \cdot \alpha^{n-m}+\frac{\alpha^{\prime}}{2} \mathfrak{m}^{2} \sum_{m>0}^{\infty} \alpha^{-m} \cdot \alpha^{n+m},
$$

the dot $\cdot$ denoting Lorentz products. Application of the canonical quantization procedure to a homogeneous invariant charge $\mathcal{Z}_{\mu_{1} \ldots \mu_{N}}^{(K)}$ as in (4.11) renders a normally ordered counterpart

$$
: \mathcal{Z}_{\mu_{1} \ldots \mu_{N}}^{(K)}:=\frac{\mathfrak{m}^{N}}{(2 \pi)^{N-K}} \frac{(-i)^{N-K}}{(K-1) !} \sum_{n_{1}=\infty}^{\infty} \cdots \sum_{n_{N}=\infty}^{\infty}: \alpha_{\mu_{1}}^{n_{1}} \cdots \alpha_{\mu_{N}}^{n_{N}} C_{n_{1} \ldots n_{N}}^{[K, N]}:
$$

The combinatorial factors are to be calculated in such a manner that after evaluation of the Kronecker symbols no indices with relative signs (for instance, $n_{1}-n_{2}$ with $n_{1}, n_{2}>0$ ) arise. This is important since we have to discriminate between positive, negative and zero modes, and the question whether an index involving a relative sign is positive, negative or zero, requires the discrimination of different cases concerning the relative magnitude of summation indices (e.g. $n_{1}>n_{2}$ in the example). A lengthy calculation yields the homogeneous invariant charges expressed in terms of annihilation and creation operators (for those needed in what follows, see appendix B).

In this section, the dimension of the underlying spacetime has so far been arbitrary. For an attempt to quantize the algebra of observables canonically, let us again specialize to $1+3$ dimensions and proceed as follows: in a first step, the normally ordered quantum analogues of the classical generators $J_{1}, T_{2}, S_{2}, S_{1}$ and $B_{0}^{(\ell)}$ are calculated by application of formula (4.14). Next, we consider the zeroth level $\mathfrak{V}^{0}$ as well as the action of $\mathfrak{V}^{0}$ on the other levels $\mathfrak{V}^{\ell}$, where the rescaled Poisson brackets are replaced by commutators multiplied by $2 \pi \alpha^{\prime} / i \hbar$. By appendix $\left[\right.$, we find for the generators of $\mathfrak{V}^{0}$ :

$$
\begin{gathered}
: J_{1,-1}:=-\frac{\mathfrak{m}^{2}}{4 \pi} \sum_{n=1}^{\infty} \frac{1}{n}\left(\alpha_{-}^{-n} \alpha_{3}^{n}-\alpha_{3}^{-n} \alpha_{-}^{n}\right), \quad: J_{1,0}:=-\frac{\mathfrak{m}^{2}}{4 \pi} \sum_{n=1}^{\infty} \frac{1}{n}\left(\alpha_{+}^{-n} \alpha_{-}^{n}-\alpha_{-}^{-n} \alpha_{+}^{n}\right), \\
: J_{1,+1}:=-\frac{\mathfrak{m}^{2}}{4 \pi} \sum_{n=1}^{\infty} \frac{1}{n}\left(\alpha_{+}^{-n} \alpha_{3}^{n}-\alpha_{3}^{-n} \alpha_{+}^{n}\right) .
\end{gathered}
$$


Obviously, commutators of the form

$$
\left[\alpha_{\mu}^{-n} \alpha_{\nu}^{n},: \text { polynomial in } \alpha \text { 's : }\right], \quad n>0,
$$

can only yield normally ordered terms, and we may conclude that the action of $J_{1, m}$ remains unchanged. Therefore, $\mathfrak{V}^{0}$ as well as the multiplet structure of $\mathfrak{V}^{\ell}$ is not affected by the quantization prescription. Regarding the quantum relations in higher degrees $\ell$, one now proceeds as follows. Write all terms of the classical relation (in terms of the appropriate multiplets) on the left hand side of an equation. Replace all generators by their normally ordered counterparts, and the rescaled Poisson brackets and products as described in section 3. The commutators are then evaluated by application of the derivation rule. Finally, all resulting terms are brought into normal order. From the classical relation it follows that in leading order the result is 0 , but from the process of reordering, quantum corrections may arise.

Explicitly, the relations which form the starting point of the calculations, are the following:

$$
\begin{aligned}
& J^{P}=4^{-} \quad: \quad \frac{2 \pi \alpha^{\prime}}{\hbar}\left[: T_{2}:,: S_{2}:\right]_{4}=0+\text { anomalies } \\
& \left.J^{P}=3^{+} i\right): \quad \frac{2 \pi \alpha^{\prime}}{\hbar}\left[: T_{2}:,: T_{2}:\right]_{3}+i \frac{2 \pi \alpha^{\prime}}{\hbar}\left[: S_{2}:,: S_{1}:\right]_{3}+16\left(: J_{1}:{ }^{3}\right)_{3}=0+\text { anomalies } \\
& \text { ii) : } \quad \frac{2 \pi \alpha^{\prime}}{\hbar}\left[: S_{2}:,: S_{2}:\right]_{3}-i 2 \frac{2 \pi \alpha^{\prime}}{\hbar}\left[: S_{2}:,: S_{1}:\right]_{3} \\
& -4\left\{: J_{1}:,: T_{2}:\right\}_{3}-48\left(: J_{1}:^{3}\right)_{3}=0+\text { anomalies } \\
& J^{P}=3^{-} \quad: \quad \frac{2 \pi \alpha^{\prime}}{\hbar}\left[: T_{2}:,: S_{2}:\right]_{3}-i \frac{2 \pi \alpha^{\prime}}{\hbar}\left[: T_{2}:,: S_{1}:\right]_{3} \\
& +4\left\{: J_{1}:,: S_{2}:\right\}_{3}=0+\text { anomalies } \\
& J^{P}=2^{-} \quad: \quad \frac{2 \pi \alpha^{\prime}}{\hbar}\left[: T_{2}:,: S_{2}:\right]_{2}+\frac{i}{3} \sqrt{\frac{7}{2}} \frac{2 \pi \alpha^{\prime}}{\hbar}\left[: T_{2}:,: S_{1}:\right]_{2} \\
& -\frac{2}{3} \sqrt{14}\left\{: J_{1}:,: S_{2}:\right\}_{2}=0+\text { anomalies } \\
& J^{P}=1^{+} \quad: \quad \frac{2 \pi \alpha^{\prime}}{\hbar}\left[: S_{2}:,: S_{2}:\right]_{1}+i \sqrt{\frac{2}{3}} \frac{2 \pi \alpha^{\prime}}{\hbar}\left[: S_{2}:,: S_{1}:\right]_{1}+\frac{1}{6} \sqrt{5} \frac{2 \pi \alpha^{\prime}}{\hbar}\left[: S_{1}:,: S_{1}:\right]_{1} \\
& -8 \sqrt{\frac{2}{3}}\left\{: J_{1}:,: T_{2}:\right\}_{1}-16 \sqrt{\frac{2}{15}}\left\{: J_{1}:,\left(: J_{1}:{ }^{2}\right)_{0}\right\}_{1} \\
& +\frac{\hbar^{2}}{\left(2 \pi \alpha^{\prime}\right)^{2}} f \sqrt{10}: J_{1}:=0+\text { anomalies }
\end{aligned}
$$

and for the action of : $B_{0}^{(1)}$ :,

$$
\begin{aligned}
& J^{P}=2^{+} \quad: \quad \frac{2 \pi \alpha^{\prime}}{\hbar}\left[: B_{0}^{(1)}:,: T_{2}:\right]_{2}-i \sqrt{6} \frac{2 \pi \alpha^{\prime}}{\hbar}\left[: S_{2}:,: S_{1}:\right]_{2}=0+\text { anomalies } \\
& J^{P}=1^{-} \quad: \quad \frac{2 \pi \alpha^{\prime}}{\hbar}\left[: B_{0}^{(1)}:,: S_{1}:\right]_{1}+i 6 \sqrt{\frac{2}{5}} \frac{2 \pi \alpha^{\prime}}{\hbar}\left[: T_{2}:,: S_{2}:\right]_{1}-2 \sqrt{\frac{3}{5}} \frac{2 \pi \alpha^{\prime}}{\hbar}\left[: T_{2}:,: S_{1}:\right]_{1} \\
& +12 i \sqrt{\frac{3}{5}}\left\{: J_{1}:,: S_{2}:\right\}_{1}+6 \sqrt{2}\left\{: J_{1}:,: S_{1}:\right\}_{1}=0+\text { anomalies } \\
& J^{P}=2^{-} \quad: \quad \frac{2 \pi \alpha^{\prime}}{\hbar}\left[: B_{0}^{(1)}:,: S_{2}:\right]_{2}+i 2 \sqrt{\frac{2}{3}} \frac{2 \pi \alpha^{\prime}}{\hbar}\left[: T_{2}:,: S_{1}:\right]_{2}-i 2 \sqrt{\frac{2}{3}}\left\{: J_{1}:,: S_{2}:\right\}_{2} \\
& -i 6\left\{: J_{1}:,: S_{1}:\right\}_{2}=0+\text { anomalies }
\end{aligned}
$$

Note that (as in the case of algebraic quantization) there is no need for the use of an anticommutator if the coupling to spin $J$ in a product $\left(: J_{1}:{ }^{n}\right)_{J}$ is unique.

The observable quantum correction $+\frac{\hbar^{2}}{\left(2 \pi \alpha^{\prime}\right)^{2}} f \sqrt{10}: J_{1}$ : found in [6] for the relation with $J^{P}=1^{+}$, was added to the left hand side of the equation (in normally ordered form) in order to simplify the comparison with the algebraically quantized relations: if they were reproduced in the canonical approach, all right hand sides would be identically 0 (with the parameter $f$ fixed). However, as we shall see below, we will find anomalies which destroy 
the algebraic structure of the algebra $\mathfrak{h}$. What is worse: the anomalies neither possess reparametrization-invariant classical counterparts, nor can they be written in terms of Virasoro generators.

\subsection{Anomalies for $\ell=2$}

Let us start with some general considerations as to which anomalies are to be expected in the relations in $\mathfrak{V}^{2}$. First we note that commutators $\left[: \mathcal{Z}_{\mu_{1} \ldots \mu_{4}}^{(2)}:,: \mathcal{Z}_{\nu_{1} \ldots \nu_{4}}^{(2)}:\right]$ yield at most $2+2-1$ annihilation operators and as many creation operators. Likewise, we find at most 3 annihilation and 3 creation operators in products $\mathfrak{m}^{-1}: \mathcal{Z}_{0 \mu_{1} \mu_{2}}^{(2)}:: \mathcal{Z}_{\nu_{1} \ldots \nu_{4}}^{(2)}$ : and $\mathfrak{m}^{-3}: \mathcal{Z}_{0 \mu_{1} \mu_{2}}^{(2)}:: \mathcal{Z}_{0 \nu_{1} \nu_{2}}^{(2)}:: \mathcal{Z}_{0 \rho_{1} \rho_{2}}^{(2)}:$, respectively (see appendix $\mathbb{B}$ ). Now, reordering terms with at most 3 annihilation and 3 creation operators, we derive a quantum correction consisting of at most 2 annihilation and 2 creation operators, and reordering such terms finally yields quantum corrections consisting of at most 1 annihilation and 1 creation operator. The expressions possess the following physical units:

\begin{tabular}{|c|c|c|c|}
\hline order & \multicolumn{3}{|c|}{ units $(\ell=2)$} \\
\hline leading $(=0)$ & $\frac{2 \pi \alpha^{\prime}}{\hbar} \frac{\mathfrak{m}^{8}}{(2 \pi)^{4}} \frac{\hbar 2 \pi}{2 \pi \alpha^{\prime} \mathfrak{m}^{2}}$ & $=$ & $\frac{\mathfrak{m}^{6}}{(2 \pi)^{3}}$ \\
\hline first reordering & $\frac{\mathfrak{m}^{6}}{(2 \pi)^{3}} \frac{\hbar 2 \pi}{2 \pi \alpha^{\prime} \mathfrak{m}^{2}}$ & $=$ & $\frac{\mathfrak{m}^{4}}{(2 \pi)^{2}} \frac{\hbar}{2 \pi \alpha^{\prime}}$ \\
\hline second reordering & $\frac{\mathfrak{m}^{4}}{(2 \pi)^{2}} \frac{\hbar}{2 \pi \alpha^{\prime}} \frac{\hbar 2 \pi}{2 \pi \alpha^{\prime} \mathfrak{m}^{2}}$ & $=$ & $\frac{\mathfrak{m}^{2}}{2 \pi} \frac{\hbar^{2}}{\left(2 \pi \alpha^{\prime}\right)^{2}}$ \\
\hline
\end{tabular}

which makes sense, as $\mathfrak{m}^{4} /(2 \pi)^{2}$ is the unit of an element of $\mathfrak{V}^{1}$, and $\mathfrak{m}^{2} /(2 \pi)$ that of an element of $\mathfrak{V}^{0}$. By the canoncial commutation relations (4.12), either two spacelike or two timelike indices are contracted, whence the parity is unchanged by reorderings. The anomalies will again arise as multiplets of $s o(3)$, such that it suffices to calculate the anomalies for fixed magnetic quantum number $m=-J$. In fact, the possible anomalies for each relation can be predicted. For example, the only possible quantum correction with $m=-4$ would be $\alpha_{-}^{(\cdot)} \alpha_{-}^{(\cdot)} \alpha_{-}^{(\cdot)} \alpha_{-}^{(\cdot)}$, which, however, has positive parity $P$ and hence cannot appear in the relation with $J^{P}=4^{-}$. While many of the possible anomalies arise somewhere in the course of the calculation, most of them cancel and only some remain in the end.

The anomalies have to be explicitly calculated, since it has to be checked whether they correspond to classical functionals which are invariant under reparametrizations, or whether at least they are functions of the Virasoro generators and as such vanish on the physical subspace. The calculations are performed for fixed but arbitrary summation indices ${ }^{3}$ and, at intermediate steps, involve several thousand terms. It is therefore necessary to use computer algebra, and the programme package Mathematica was employed (for an explanation of the devised routines see [1]). In the course of the calculation, some simplifications

\footnotetext{
${ }^{3}$ The convergence problem of the infinite series of operators is ignored, since the sole purpose of this investigation is the comparison with the ordinary canonical approach, where these questions likewise do not play a role.
} 
have to be done by hand, such as

$$
\begin{aligned}
& -\sum_{n_{1}, n_{2}>0} \frac{3}{n_{1} n_{2}} \alpha_{\mu}^{-n_{1}-n_{2}} \alpha_{\nu}^{n_{1}} \alpha_{\nu}^{n_{2}}+\sum_{n_{1}, n_{2}>0} \frac{4}{n_{1}\left(n_{1}+n_{2}\right)} \alpha_{\mu}^{-n_{1}-n_{2}} \alpha_{\nu}^{n_{1}} \alpha_{\nu}^{n_{2}} \\
& +\sum_{n_{1}, n_{2}>0} \frac{2}{n_{2}\left(n_{1}+n_{2}\right)} \alpha_{\mu}^{-n_{1}-n_{2}} \alpha_{\nu}^{n_{1}} \alpha_{\nu}^{n_{2}}=\sum_{n_{1}, n_{2}>0} \frac{-n_{1}+n_{2}}{n_{1} n_{2}\left(n_{1}+n_{2}\right)} \alpha_{\mu}^{-n_{1}-n_{2}} \alpha_{\nu}^{n_{1}} \alpha_{\nu}^{n_{2}}=0,
\end{aligned}
$$

or

$$
\sum_{n_{1}, n_{2}>0} \frac{1}{n_{1} n_{2}} \alpha_{\mu}^{-n_{1}-n_{2}} \alpha_{\nu}^{n_{1}} \alpha_{\nu}^{n_{2}}=\sum_{n_{1}, n_{2}>0} \frac{2}{n_{1}\left(n_{1}+n_{2}\right)} \alpha_{\mu}^{-n_{1}-n_{2}} \alpha_{\nu}^{n_{1}} \alpha_{\nu}^{n_{2}} .
$$

\begin{tabular}{|c|c|}
\hline$J^{P}$ & anomalies for $m=-J$ \\
\hline $4^{-}$ & 0 \\
\hline $\left.3^{+} \quad i\right)$ & $\frac{\hbar}{2 \pi \alpha^{\prime}} \frac{\mathfrak{m}^{4}}{(2 \pi)^{2}} \sum_{n, m>0} \frac{-8}{n m}\left(\alpha_{-}^{-n} \alpha_{-}^{-m} \alpha_{-}^{m} \alpha_{3}^{n}-\alpha_{3}^{-n} \alpha_{-}^{-m} \alpha_{-}^{m} \alpha_{-}^{n}\right)$ \\
\hline$i i)$ & $\frac{\hbar}{2 \pi \alpha^{\prime}} \frac{\mathfrak{m}^{4}}{(2 \pi)^{2}} \sum_{n, m>0} \frac{20}{n m}\left(\alpha_{-}^{-n} \alpha_{-}^{-m} \alpha_{-}^{m} \alpha_{3}^{n}-\alpha_{3}^{-n} \alpha_{-}^{-m} \alpha_{-}^{m} \alpha_{-}^{n}\right)$ \\
\hline $3^{-}$ & $\begin{array}{r}\frac{\hbar}{2 \pi \alpha^{\prime}} \frac{\mathfrak{m}^{4}}{(2 \pi)^{2}} \sum_{n, m>0} \frac{4 i}{n m}\left(2 \alpha_{0}^{-n} \alpha_{-}^{-m} \alpha_{-}^{m} \alpha_{-}^{n}-2 \alpha_{-}^{-n} \alpha_{-}^{-m} \alpha_{-}^{m} \alpha_{0}^{n}\right. \\
\left.+\alpha_{-}^{-n} \alpha_{-}^{-m} \alpha_{-}^{n+m}-\alpha_{-}^{-n-m} \alpha_{-}^{n} \alpha_{-}^{m}\right)\end{array}$ \\
\hline $2^{-}$ & 0 \\
\hline $1^{+}$ & $\begin{aligned} \frac{\hbar}{2 \pi \alpha^{\prime}} \frac{\mathfrak{m}^{4}}{(2 \pi)^{2}} \frac{1}{\sqrt{10}} & \frac{80}{3}\left(\sum_{n>0} \frac{1}{n^{2}}\left(\alpha_{3}^{-n} \alpha_{-}^{n}-\alpha_{-}^{-n} \alpha_{3}^{n}\right)\right. \\
& \left.+\sum_{n, m>0} \frac{1}{(n+m)^{2}}\left(\alpha_{3}^{-n-m} \alpha_{-}^{n+m}-\alpha_{-}^{-n-m} \alpha_{3}^{n+m}\right)\right)\end{aligned}$ \\
\hline
\end{tabular}

In order to check the manipulations, it was calculated that indeed, the leading order terms in the relations yield 0 . The results of the calculation are:

and similar results were found for the relations involving $B_{0}^{(1)}$ (see appendix $\mathbf{C}$ ). In addition to the anomalies given above, the following term appeared in the relation with $J^{P}=1^{+}$ for $m=-1$,

$$
\frac{\hbar^{2}}{\left(2 \pi \alpha^{\prime}\right)^{2}} \frac{\mathfrak{m}^{2}}{2 \pi} \frac{1}{\sqrt{10}} \frac{64}{3} \sum_{n>0} \frac{1}{n}\left(\alpha_{3}^{-n} \alpha_{-}^{n}-\alpha_{-}^{-n} \alpha_{3}^{n}\right),
$$

whence we deduce that $f=-\frac{128}{30}$. Note that this is not consistent with the result found later by the method presented in [10].

The anomalies in the relation with $J^{P}=1^{+}$can be rewritten in the following way. Since there are $n-1$ possibilities to write $\mathbb{N} \ni n>0$ as a sum of two natural numbers $n_{i}>0$, the following identity holds:

$$
\sum_{n_{1}, n_{2}>0} \frac{1}{\left(n_{1}+n_{2}\right)^{2}} X_{\left(n_{1}+n_{2}\right)}=\sum_{n>0} \frac{n-1}{n^{2}} X_{n},
$$

and the anomalies for $J^{P}=1^{+}$can be simplified to yield

$$
\begin{gathered}
\frac{\hbar}{2 \pi \alpha^{\prime}} \frac{\mathfrak{m}^{4}}{(2 \pi)^{2}}\left(\sum_{n>0} \frac{1}{n^{2}}\left(\alpha_{3}^{-n} \alpha_{-}^{n}-\alpha_{-}^{-n} \alpha_{3}^{n}\right)+\sum_{n>0}\left(\frac{1}{n}-\frac{1}{n^{2}}\right)\left(\alpha_{3}^{-n} \alpha_{-}^{n}-\alpha_{-}^{-n} \alpha_{3}^{n}\right)\right) \\
\quad=\frac{\hbar}{2 \pi \alpha^{\prime}} \frac{\mathfrak{m}^{4}}{(2 \pi)^{2}} \sum_{n>0} \frac{1}{n}\left(\alpha_{3}^{-n} \alpha_{-}^{n}-\alpha_{-}^{-n} \alpha_{3}^{n}\right)=\frac{\hbar}{2 \pi \alpha^{\prime}} \frac{\mathfrak{m}^{2}}{\pi}: J_{1,-1}: .
\end{gathered}
$$


At first sight, this is surprising, since after once reordering an expression in $\mathfrak{V}^{2}$ we would not expect an element of $\mathfrak{V}^{0}$ to arise (but rather one of $\mathfrak{V}^{1}$ ). However, as was remarked on page 8, the tensor rank of an expression in terms of annihilation and creation operators (or Fourier components) is given by the power of $\mathfrak{m}$ in the expression, while the corresponding degree $\ell$ is given only by the negative power of $2 \pi$ in the expression minus 1 (which is correctly given here by $\ell=2-1$ ). We should therefore not think of the above expression as an element of $\mathfrak{V}^{0}$, but rather as an anomaly with $\ell=1$, accidentally having a similar form as $J_{1,-1} \in \mathfrak{V}^{0}$. It is illustrative to explicitly retrace how this term arises.

Remark 1 The term (4.15) is part of the following commutator:

$$
\frac{2 \pi \alpha^{\prime}}{\hbar}\left[: \mathcal{Z}_{0-++}^{(2)}:,: \mathcal{Z}_{03--}^{(2)}:\right]
$$

which appears in the relation with $J^{P}=1^{+}, m=-1$.

Proof: First note that by (2.7) this commutator appears in $\left[S_{2}, S_{1}\right]_{1,-1}$ and hence, is indeed part of the relation under consideration. Now, in the homogeneous invariants $: \mathcal{Z}_{\mu_{1} \ldots \mu_{4}}^{(2)}:$, terms of the following form appear,

$$
\frac{\mathfrak{m}^{4}}{(2 \pi)^{2}} \delta_{\mu_{1}, 0} \sum_{n_{1}>0} \sum_{n_{2}>0} \frac{1}{n_{1}\left(n_{1}+n_{2}\right)}\left(\alpha_{\mu_{2}}^{-n_{1}-n_{2}} \alpha_{\mu_{4}}^{n_{1}} \alpha_{\mu_{3}}^{n_{2}}+\alpha_{\mu_{4}}^{-n_{1}} \alpha_{\mu_{3}}^{-n_{2}} \alpha_{\mu_{2}}^{n_{1}+n_{2}}\right)
$$

such that a commutator $\frac{2 \pi \alpha^{\prime}}{\hbar}\left[: \mathcal{Z}_{\mu_{1} \ldots \mu_{4}}^{(2)}:,: \mathcal{Z}_{\nu_{1} \ldots \nu_{4}}^{(2)}:\right]$ yields (among other terms)

$$
\begin{aligned}
& \frac{\mathfrak{m}^{8}}{(2 \pi)^{4}} \frac{2 \pi \alpha^{\prime}}{\hbar} \sum_{n_{1}, n_{2}>0} \sum_{m_{1}, m_{2}>0} \frac{1}{n_{1}\left(n_{1}+n_{2}\right) m_{1}\left(m_{1}+m_{2}\right)} \alpha_{\mu_{2}}^{-n_{1}-n_{2}} \alpha_{\mu_{4}}^{n_{1}}\left[\alpha_{\mu_{3}}^{n_{2}}, \alpha_{\nu_{4}}^{-m_{1}}\right] \alpha_{\nu_{3}}^{-m_{2}} \alpha_{\nu_{2}}^{m_{1}+m_{2}} \\
& \quad=\frac{2 \mathfrak{m}^{6}}{(2 \pi)^{3}} \sum_{n_{1}, n_{2}>0} \sum_{m_{2}>0} \frac{\eta_{\mu_{3} \nu_{4}}}{n_{1}\left(n_{1}+n_{2}\right)\left(n_{2}+m_{2}\right)} \alpha_{\mu_{2}}^{-n_{1}-n_{2}} \alpha_{\mu_{4}}^{n_{1}} \alpha_{\nu_{3}}^{-m_{2}} \alpha_{\nu_{2}}^{n_{2}+m_{2}} .
\end{aligned}
$$

Hence from (4.16) we find the contribution

$$
-\frac{\mathfrak{m}^{6}}{4 \pi^{3}} \sum_{n_{1}, n_{2}, m_{2}>0} \frac{1}{n_{1}\left(n_{1}+n_{2}\right)\left(n_{2}+m_{2}\right)} \alpha_{-}^{-n_{1}-n_{2}} \alpha_{+}^{n_{1}} \alpha_{-}^{-m_{2}} \alpha_{3}^{n_{2}+m_{2}}
$$

which by normal ordering yields the anomaly

$$
\begin{aligned}
& -\frac{\mathfrak{m}^{6}}{4 \pi^{3}} \sum_{n_{1}, n_{2}, m_{2}>0} \frac{1}{n_{1}\left(n_{1}+n_{2}\right)\left(n_{2}+m_{2}\right)}\left[\alpha_{+}^{n_{1}}, \alpha_{-}^{-m_{2}}\right] \alpha_{-}^{-n_{1}-n_{2}} \alpha_{3}^{n_{2}+m_{2}} \\
& =\frac{\hbar}{2 \pi \alpha^{\prime}} \frac{\mathfrak{m}^{4}}{\pi^{2}} \sum_{n_{1}, n_{2}>0} \frac{1}{\left(n_{1}+n_{2}\right)^{2}} \alpha_{-}^{-n_{1}-n_{2}} \alpha_{3}^{n_{1}+n_{2}}
\end{aligned}
$$

and in the same manner we find $-\frac{\hbar}{2 \pi \alpha^{\prime}} \frac{\mathfrak{m}^{4}}{\pi^{2}} \sum_{n_{1}, n_{2}>0} \frac{1}{\left(n_{1}+n_{2}\right)^{2}} \alpha_{3}^{-n_{1}-n_{2}} \alpha_{-}^{n_{1}+n_{2}}$.

Let us now turn to an interpretation of the anomalies.

\subsection{Unobservability of the anomalies}

First we note that in the conformal gauge (4.9) the anomalies are independent of $\tau$.

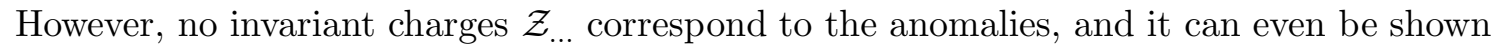
directly that the anomalies do not in general correspond to classical functionals on the world-sheet which are invariant under changes of the parametrization. To see this, we rewrite the anomalies in a more compact manner as multiplets of the $s o(3)$. To that end, the following operators are defined, 
i.) $: R_{1}:=\left\{: R_{1,-1}:,: R_{1,0}:,: R_{1,1}:\right\} \stackrel{\text { def }}{=}\left\{: R_{0-}:,: R_{03}:,-: R_{0+}:\right\}, \quad$ with

$$
\begin{gathered}
\mathcal{M}_{\mu \nu}=x_{\mu} \mathcal{P}_{\nu}-x_{\nu} \mathcal{P}_{\mu}+\frac{2 \pi \alpha^{\prime}}{2 i} \underbrace{\frac{\mathfrak{m}^{2}}{2 \pi} \sum_{n>0} \frac{1}{n}\left(\alpha_{\mu}^{-n} \alpha_{\nu}^{n}-\alpha_{\nu}^{-n} \alpha_{\mu}^{n}\right.}_{=: R_{\mu \nu}:}+\beta_{\mu}^{-n} \beta_{\nu}^{n}-\beta_{\nu}^{-n} \beta_{\mu}^{n}) . \\
\text { ii. }): A_{2, m}:=\frac{\mathfrak{m}^{2}}{2 \pi} \sum_{n>0} \frac{1}{n}\left(\alpha_{1}^{-n} \alpha_{1}^{n}\right)_{2, m}=\frac{\mathfrak{m}^{2}}{2 \pi} \sum_{n>0} \frac{1}{n} \underbrace{\sum_{m_{1}}^{1}}_{\underbrace{m_{1}, m_{2}=-1}_{m_{1}+m_{2}=m}}\left\langle 2, m \mid 1, m_{1} ; 1, m_{2}\right\rangle \alpha_{1, m_{1}}^{-n} \alpha_{1, m_{2}}^{n}
\end{gathered}
$$

with $\alpha_{1,-1}^{ \pm n}=\alpha_{-}^{ \pm n}, \alpha_{1,0}^{ \pm n}=\alpha_{3}^{ \pm n}$ and $\alpha_{1,1}^{ \pm n}=-\alpha_{+}^{ \pm n}$.

: $A_{2, m}$ : is symmetric in the coordinate indices, since for the Clebsch-Gordan coefficients we have: $\langle 2, \pm 1 \mid 1, \pm 1 ; 1,0\rangle=\langle 2, \pm 1 \mid 1,0 ; 1, \pm 1\rangle$ and $\langle 2,0 \mid 1,-1 ; 1,1\rangle=\langle 2,0 \mid 1,1 ; 1,-1\rangle$.

\begin{tabular}{|c|c|c|}
\hline$J^{P}$ & anomalies & \\
\hline $4^{-}$ & 0 & \\
\hline $\left.3^{+} \quad i\right)$ & $\frac{\hbar}{2 \pi \alpha^{\prime}} 8\left\{: J_{1}:,: A_{2}:\right\}_{3}$ & \\
\hline$i i)$ & $-\frac{\hbar}{2 \pi \alpha^{\prime}} 20\left\{: J_{1}:,: A_{2}:\right\}_{3}$ & \\
\hline $3^{-}$ & $\frac{\hbar}{2 \pi \alpha^{\prime}} 4 i\left\{: R_{1}:,: A_{2}:\right\}_{3}$ & $+\quad$ "more" \\
\hline $2^{-}$ & 0 & \\
\hline $1^{+}$ & $\frac{\hbar}{2 \pi \alpha^{\prime}} \frac{1}{\sqrt{10}} \frac{80}{3} \frac{\mathfrak{m}^{2}}{\pi}: J_{1}:$ & \\
\hline
\end{tabular}

An elementary calculation now shows that the anomalies can be written as follows

with anticommutators $\{\cdot, \cdot\}_{j, m}$ again coupled to spin $j$ and magnetic quantum number $m$, and where (still) $f=-\frac{128}{30}$. Here, the term "more" in a relation indicates that further anomalous terms appear which involve mixed summation indices such as $n+m$ and for which no further simplification has been found. Similarly, for the relations involving $B_{0}^{(1)}$,

\begin{tabular}{l|cc}
$J^{P}$ & anomalies & \\
\hline $2^{+}$ & $-\frac{\hbar}{2 \pi \alpha^{\prime}} 4 \sqrt{6}\left\{: J_{1}:,: A_{2}:\right\}_{2}$ & \\
$2^{-}$ & $-\frac{\hbar}{2 \pi \alpha^{\prime}} 2 \sqrt{6} i\left\{: R_{1}:,: A_{2}:\right\}_{2} \quad+\quad$ "more" \\
$1^{-}$ & $-\frac{\hbar}{2 \pi \alpha^{\prime}} 12 \sqrt{\frac{3}{5}}\left\{: R_{1}:,: A_{2}:\right\}_{1} \quad+\quad$ "more" \\
& $+\frac{\hbar^{2}}{\left(2 \pi \alpha^{\prime}\right)^{2}} 12: R_{1}:$
\end{tabular}

where the anomaly of second order in the relation with $J^{P}=1^{-}$appears when the normally ordered anomaly term is written as the anticommutator $\left\{: R_{1}:,: A_{2}:\right\}_{1}$.

We are now prepared to state the main result of the present investigation. 
Remark 2 The classical symmetric monomials $A_{2, m}$ which correspond to the products $: A_{2, m}$ : are not observable.

Proof: Consider a left mover $u_{\mu}$ which is written in terms of classical Fourier modes (4.8) and split it into its negative, positive and null modes,

$$
u_{\mu}(\sigma)=\sum_{n>0} \alpha_{\mu}^{n} e^{i n \sigma}+\sum_{n>0} \alpha_{\mu}^{-n} e^{-i n \sigma}+\alpha_{\mu}^{0}=: u_{\mu}^{p}(\sigma)+u_{\mu}^{n}(\sigma)+\alpha_{\mu}^{0},
$$

and calculate the following integral, which is symmetrized in the coordinate indices $\mu$ and $\nu$,

$$
\begin{aligned}
& I(\mu, \nu)=\int_{0}^{2 \pi} d \sigma_{1} u_{(\mu}\left(\sigma_{1}\right) \int_{0}^{\sigma_{1}} d \sigma_{2}\left(u_{\nu)}^{p}\left(\sigma_{2}\right)-u_{\nu)}^{n}\left(\sigma_{2}\right)\right) \\
& =\frac{\mathfrak{m}^{2}}{4 \pi^{2}} \int_{0}^{2 \pi} d \sigma_{1} \sum_{n_{1}=-\infty}^{\infty} e^{i n_{1} \sigma_{1}} \alpha_{(\mu}^{n_{1}}\left(\sum_{n_{2}>0} \alpha_{\nu)}^{n_{2}} \frac{e^{i n_{2} \sigma_{1}}-1}{i n_{2}}-\sum_{n_{2}>0} \alpha_{\nu)}^{-n_{2}} \frac{e^{-i n_{2} \sigma_{1}}-1}{-i n_{2}}\right) \\
& =\frac{\mathfrak{m}^{2}}{4 \pi^{2}} \sum_{n_{1}=-\infty}^{\infty} \sum_{n_{2}>0} \alpha_{(\mu}^{n_{1}} \alpha_{\nu)}^{n_{2}} \frac{1}{i n_{2}} 2 \pi\left(\delta_{n_{1}+n_{2}, 0}-\delta_{n_{1}, 0}\right) \\
& +\frac{\mathfrak{m}^{2}}{4 \pi^{2}} \sum_{n_{1}=-\infty}^{\infty} \sum_{n_{2}>0} \alpha_{(\mu}^{n_{1}} \alpha_{\nu)}^{-n_{2}} \frac{1}{i n_{2}} 2 \pi\left(\delta_{n_{1}-n_{2}, 0}-\delta_{n_{1}, 0}\right) \\
& =\frac{\mathfrak{m}^{2}}{2 \pi} \frac{2}{i} \sum_{n_{2}>0} \frac{1}{n_{2}} \alpha_{(\mu}^{-n_{2}} \alpha_{\nu)}^{n_{2}}-\underbrace{\frac{\mathfrak{m}^{2}}{4 \pi^{2}} \frac{2 \pi}{i} \sum_{n_{2}>0} \frac{1}{n_{2}}\left(\alpha_{(\mu}^{0} \alpha_{\nu)}^{n_{2}}+\alpha_{(\mu}^{0} \alpha_{\nu)}^{-n_{2}}\right)}_{\circledast} .
\end{aligned}
$$

For $\mu$ and $\nu$ spacelike, the term $\circledast$ vanishes in the rest system and hence, the products : $A_{2, m}$ : (which contain spacelike indices only) are linear combinations of integrals of the form $I(\mu, \nu)$. Now consider a change of parametrization $\sigma \rightarrow \hat{\sigma}$, then the integral over $u_{\nu}$ is invariant,

$$
\int_{\sigma_{0}}^{\sigma} d \sigma_{1}\left[u_{\nu}^{p}\left(\sigma_{1}\right)+u_{\nu}^{n}\left(\sigma_{1}\right)+\alpha_{\nu}^{0}\right]=\int_{\sigma_{0}}^{\sigma} d \sigma_{1} u_{\nu}\left(\sigma_{1}\right)=\int_{\hat{\sigma}_{o}}^{\hat{\sigma}} d \hat{\sigma}_{1} \hat{u}_{\nu}\left(\hat{\sigma}_{1}\right)=\int_{\hat{\sigma}_{0}}^{\hat{\sigma}} d \hat{\sigma}_{1}\left[\hat{u}_{\nu}^{p}\left(\hat{\sigma}_{1}\right)+\hat{u}_{\nu}^{n}\left(\hat{\sigma}_{1}\right)+\alpha_{\nu}^{0}\right] .
$$

Here, we have used that the zero modes $\alpha_{\nu}^{0}$ are independent of the parametrization. Note that the splitting of $\hat{u}$ into positive and negative modes has to be done with respect to the new parametrization. In contrast to this, the difference of positive and negative modes does not in general possess the correct behaviour under reparametrizations,

$$
\int_{\sigma_{0}}^{\sigma} d \sigma_{1}\left[u_{\nu}^{p}\left(\sigma_{1}\right)-u_{\nu}^{n}\left(\sigma_{1}\right)\right] \stackrel{i . g .}{\neq} \int_{\hat{\sigma}_{0}}^{\hat{\sigma}} d \hat{\sigma}_{1}\left[\hat{u}_{\nu}^{p}\left(\hat{\sigma}_{1}\right)-\hat{u}_{\nu}^{n}\left(\hat{\sigma}_{1}\right)\right] .
$$

To see this, consider the following counter example. Given a left mover with

$$
u_{\mu}(\sigma)=1 \delta_{\mu, 0}=\alpha_{\mu}^{0} \delta_{\mu, 0}, \quad \text { hence } u_{\nu}^{p}(\sigma)=u_{\nu}^{n}(\sigma)=0,
$$

the left hand side of (4.17) is zero, while this is not true in general for the right hand side. Consider the reparametrization $\sigma \rightarrow \hat{\sigma}$ with $\sigma=f(\hat{\sigma})$, where

$$
e^{i \sigma}=\frac{e^{i \hat{\sigma}}-w}{\bar{w} e^{i \hat{\sigma}}-1} \quad \text { with fixed } w, \quad|w|<1, \quad w=|w| e^{i \chi} .
$$

In more technical terms, consider the unit disk $\subset \mathbb{C}$, whose boundary corresponds to the string, then the above defines an automorphism of the disk which maps the boundary of the disk to itself, 
while respecting its orientation. Therefore, it does indeed define a parametrization. Obviously, we have

$$
d \sigma=\left(1-|w|^{2}\right) \frac{d \hat{\sigma}}{\left(1-\bar{w} e^{i \hat{\sigma}}\right)\left(1-w e^{-i \hat{\sigma}}\right)},
$$

and hence, the transformed left mover $\hat{u}_{0}(\hat{\sigma})$ is given as (the other components, $u_{\mu \neq 0}$, vanish):

$$
\hat{u}_{0}(\hat{\sigma})=u_{0}(f(\hat{\sigma})) \frac{d f(\hat{\sigma})}{d \hat{\sigma}}=\frac{1-|w|^{2}}{\left(1-\bar{w} e^{i \hat{\sigma}}\right)\left(1-w e^{-i \hat{\sigma}}\right)} .
$$

In particular, the zero mode $\hat{u}_{0}^{0}(\hat{\sigma})=u_{0}^{0}(\sigma)=1$ is invariant, while for positive and negative modes an explicit calculation yields

$$
\begin{aligned}
& \hat{u}_{0}^{p}(\hat{\sigma})=\left(1-|w|^{2}\right) \sum_{l \geq 1}\left(\sum_{n \geq 0}|w|^{2 n}\right)|w|^{l} e^{-i l \chi} e^{i l \hat{\sigma}} \\
& \hat{u}_{0}^{n}(\hat{\sigma})=\left(1-|w|^{2}\right) \sum_{l \leq-1}\left(\sum_{n \geq|l|}|w|^{2 n}\right)|w|^{l} e^{-i l \chi} e^{i l \hat{\sigma}} .
\end{aligned}
$$

We may thus conclude that while the left hand side of (4.17) is zero, the right hand side yields

$$
\begin{aligned}
\int_{\hat{\sigma}_{0}}^{\hat{\sigma}} d \hat{\sigma}_{1}\left[\hat{u}_{\nu}^{p}\left(\hat{\sigma}_{1}\right)-\hat{u}_{\nu}^{n}\left(\hat{\sigma}_{1}\right)\right]=\delta_{\nu, 0}\left(1-|w|^{2}\right) \sum_{l \geq 1} & \left(\sum_{n \geq 0}|w|^{2 n}|w|^{l} e^{-i l \chi} \frac{e^{i l \hat{\sigma}}-e^{i l \hat{\sigma}_{0}}}{i l}\right. \\
& \left.-\sum_{n \geq l}|w|^{2 n}|w|^{-l} e^{i l \chi} \frac{e^{-i l \hat{\sigma}}-e^{-i l \hat{\sigma}_{0}}}{-i l}\right) \neq 0
\end{aligned}
$$

for general $\hat{\sigma} \neq \hat{\sigma}_{0}+2 \pi$. Therefore, the products $A_{2, m}$ are not invariant under general reparametrizations.

An alternative proof of the above remark is to show that the classical monomial corresponding to an anomaly does not Poisson-commute with the generators of the Witt algebra. For instance, we find for the Poisson bracket of $A_{2,-2}=\frac{\mathfrak{m}^{2}}{2 \pi} \sum \frac{1}{n} \alpha_{-}^{-n} \alpha_{-}^{n}$ and $\bar{L}^{l}$, $l \geq 2$, the following term,

$$
-\sum_{m=1}^{l-1} \alpha_{-}^{m} \alpha_{-}^{l-m}
$$

and similarly for $l \leq-2$. Since the anomalies cannot be written as functions of the constraints, they do not even vanish weakly (on the physical subspace) and we may conclude that not even in this sense, the invariant charges in $3+1$ dimensions can be represented as a subalgebra of the polynomial algebra of normally ordered annihilation and creation operators.

To conclude, it is emphasized that the anomalies are not multiples of the identity, and therefore, canonical quantization does not merely yield a central extension of the algebra of invariant charges.

\section{Anomalies appear in any dimension}

The calculations in the preceeding section were performed in a $d=3+1$ dimensional background. A natural objection would be to claim that in some critical dimension, the problem could be absent. This, however, is not the case. 
From the relation in $d=3+1$ with $J^{P}=2^{+}$involving the exceptional element $B_{0}^{(1)}$, we can deduce that in arbitrary dimensions $d$, commutators of normally ordered invariant charges will in general yield anomalies which do not correspond to classical reparametrizationinvariant quantities. This follows directly from the fact that the relation under consideration for say $m=-2$ can also be read independently of the dimension $d$ as follows,

$$
\begin{aligned}
& \frac{1}{2}\left[: \mathcal{Z}_{0-0+}^{(2)}:+\frac{1}{2}: \mathcal{Z}_{0303}^{(2)}:,: \mathcal{Z}_{00--}^{(2)}:\right]+\left[: \mathcal{Z}_{0-33}^{(2)}:,: \mathcal{Z}_{0-33}^{(2)}:\right]-\left[: \mathcal{Z}_{0-33}^{(2)}:,: \mathcal{Z}_{0+--}^{(2)}:\right] \\
& +\left[: \mathcal{Z}_{0+--}^{(2)}:,: \mathcal{Z}_{0-33}^{(2)}:\right]-\left[: \mathcal{Z}_{0+--}^{(2)}:,: \mathcal{Z}_{0+--}^{(2)}:\right]-2\left[: \mathcal{Z}_{03--}^{(2)}:,: \mathcal{Z}_{0-3+}^{(2)}:\right] \\
& =-\frac{\hbar}{2 \pi \alpha^{\prime}} 8\left(+\frac{\sqrt{2}}{4 \mathfrak{m}}\left\{i: \mathcal{Z}_{0-3}^{(2)}:-i: \mathcal{Z}_{03-}^{(2)}:, X\right\}-\frac{1}{2 \mathfrak{m}}\left\{i: \mathcal{Z}_{0+-}^{(2)}:-i: \mathcal{Z}_{0-+}^{(2)}:, Y\right\}\right),
\end{aligned}
$$

with

$$
X=\frac{\mathfrak{m}^{2}}{2 \pi} \frac{1}{\sqrt{2}} \sum_{n>0} \frac{1}{n}\left(\alpha_{-}^{-n} \alpha_{3}^{n}+\alpha_{3}^{-n} \alpha_{-}^{n}\right), \quad Y=\frac{\mathfrak{m}^{2}}{2 \pi} \sum_{n>0} \frac{1}{n} \alpha_{-}^{-n} \alpha_{-}^{n},
$$

and where the basis $e_{0}, e_{ \pm}=\frac{1}{\sqrt{2}}\left(e_{1} \pm i e_{2}\right), e_{3}, \ldots e_{d-1}$ is chosen in $d$-dimensional Minkowski space.

Although the above may not be one of the defining relations in $d$ dimensions, its classical counterpart (where the commutators are again replaced by Poisson brackets and the right hand side is set to 0 ) is an identity in the Poisson algebra of invariant charges for arbitrary dimension $d$. The proof of Remark 2 being independent of the dimension of the underlying space, we deduce that the right hand side of the above still is not observable. Neither is it a function of the Virasoro generators, and hence the algebra of normally ordered invariant charges is no subalgebra of the normally ordered polynomials in annhihilation and creation operators, not even on the physical subspace and independently of the dimension $d$.

It is instructive to consider this result also from the following different point of view.

Remark 3 Consider the commutator of a normally ordered invariant charge $: \mathcal{Z}_{\mu_{1} \ldots \mu_{4}}^{(2)}$ : with a Virasoro generator : $\bar{L}^{n}:, n>0$. Then from the terms in $: \bar{L}^{n}$ : which involve two annihilation operators, we find anomalies of the following form (in the Cartesian basis $\left.e_{0}, e_{1}, e_{2}, \ldots, e_{d-1}\right)$ :

$$
\begin{aligned}
A\left(i_{1}, i_{2}, i_{3}, i_{4}\right) & =\eta_{\mu_{i_{1}} \mu_{i_{2}}} \sum_{n_{1}=1}^{n-1}\left(\alpha_{\mu_{i_{3}}}^{n-n_{1}} \alpha_{\mu_{i_{4}}}^{n_{1}}+\alpha_{\mu_{i_{3}}}^{n_{1}} \alpha_{\mu_{i_{4}}}^{n-n_{1}}\right), \\
A\left(i_{1}, i_{2}, i_{3}\right) & =\eta_{\mu_{i_{1}} \mu_{i_{2}}} \frac{1}{2} n(n-1) \alpha_{\mu_{i_{3}}}^{n} .
\end{aligned}
$$

Proof: The claim follows from appendix B by simple calculations. Anomalies of the form (5.18) arise from normal ordering expressions such as

$$
\begin{aligned}
& \sum_{m_{1}, m_{2}>0} \frac{1}{m_{1} m_{2}} \sum_{n_{1}=1}^{n-1} \sum_{\nu=0}^{d-1}\left[\alpha_{\nu}^{n-n_{1}}, \alpha_{\mu_{i_{1}}}^{-m_{1}}\right] \alpha^{n_{1} \nu} \alpha_{\mu_{i_{2}}}^{-m_{2}} \alpha_{\mu_{i_{3}}}^{m_{2}} \alpha_{\mu_{i_{4}}}^{m_{1}} \\
& +\sum_{m_{1}, m_{2}>0} \frac{1}{m_{1} m_{2}} \sum_{n_{1}=1}^{n-1} \sum_{\nu=0}^{d-1}\left[\alpha_{\nu}^{n-n_{1}}, \alpha_{\mu_{i_{2}}}^{-m_{2}}\right] \alpha^{n_{1} \nu} \alpha_{\mu_{i_{1}}}^{-m_{1}} \alpha_{\mu_{i_{3}}}^{m_{2}} \alpha_{\mu_{i_{4}}}^{m_{1}}
\end{aligned}
$$

Likewise, anomalies of the form (5.19) arise from normal ordering of expressions

$$
\sum_{m_{1}, m_{2}>0} \frac{1}{m_{1}\left(m_{1}+m_{2}\right)} \sum_{n_{1}=1}^{n-1} \sum_{\nu=0}^{d-1} \alpha^{n_{1} \nu}\left[\alpha_{\nu}^{n-n_{1}}, \alpha_{\mu_{i_{1}}}^{-m_{1}} \alpha_{\mu_{i_{2}}}^{-m_{2}}\right] \alpha_{\mu_{i_{3}}}^{m_{1}+m_{2}}
$$


as well as

$$
\sum_{m_{1}, m_{2}>0} \frac{1}{m_{1} m_{2}} \sum_{n_{1}=1}^{n-1} \sum_{\nu=0}^{d-1} \alpha^{n_{1} \nu}\left[\alpha_{\nu}^{n-n_{1}}, \alpha_{\mu_{i_{1}}}^{-m_{1}} \alpha_{\mu_{i_{2}}}^{-m_{2}}\right] \alpha_{\mu_{i_{3}}}^{m_{1}+m_{2}}
$$

Here, the anomalies turn out to be independent of $n_{1}$ such that the sum $\sum_{n_{1}=1}^{n-1}$ yields the factor $\frac{1}{2} n(n-1)$.

No other anomalies appear, since one needs at least 3 operators which are not multiples of the identity and at least two creation operators in order to find nontrivial contributions. The leading order of the commutator is still 0 , as it corresponds to the classical result (the invariant charges Poisson-commute with the generators of the Witt algebra). From appendix B it can then be calculated that the complete set of anomalies is given by:

$$
\begin{array}{r}
A(1,2,3,4)-A(1,3,4,2)-A(1,3,2,4)+A(1,4,3,2) \\
+A(2,3,4,1)-A(2,4,3,1)-A(2,4,1,3)+A(3,4,1,2)
\end{array}
$$

and

$$
\begin{aligned}
& -\left(\delta_{\mu_{1}, 0} A(2,3,4)+\delta_{\mu_{1}, 0} A(4,3,2)+\delta_{\mu_{2}, 0} A(3,4,1)+\delta_{\mu_{2}, 0} A(1,4,3)\right. \\
& \left.+\delta_{\mu_{3}, 0} A(4,1,2)+\delta_{\mu_{3}, 0} A(2,1,4)+\delta_{\mu_{4}, 0} A(1,2,3)+\delta_{\mu_{4}, 0} A(3,2,1)\right) \\
& +\left(\delta_{\mu_{1}, 0} A(2,4,3)+\delta_{\mu_{2}, 0} A(1,3,4)+\delta_{\mu_{3}, 0} A(2,4,1)+\delta_{\mu_{4}, 0} A(1,3,2)\right) .
\end{aligned}
$$

These anomalies do not vanish by choosing a particular dimension of the underlying Minkowski space. Neither are they functions of the Virasoro generators and hence they do not vanish on the physical subspace. To see that they furthermore do not in general correspond to classical observables, we consider the following example in $d$ dimensions with canonical basis $e_{0}, \ldots, e_{d-1}$,

$$
\left[: \bar{L}^{m}:,: \mathcal{Z}_{0011}:\right], \quad m \geq 2 .
$$

Here, a simple calculation shows that the anomalies are proportional to

$$
2 \sum_{n=1}^{m-1}\left(\alpha_{1}^{m-n} \alpha_{1}^{n}-\alpha_{0}^{m-n} \alpha_{0}^{n}\right) \quad \text { and } \quad m(m-1)\left(\alpha_{0}^{m}-\alpha_{1}^{m}\right),
$$

such that the Poisson bracket of $\bar{L}^{l}$ with the classical momomials corresponding to (5.20) yields terms of the form

$$
\begin{aligned}
& 2 \sum_{n=1}^{m-1}\left((m-n)\left(\alpha_{1}^{n} \alpha_{1}^{l+m-n}-\alpha_{0}^{n} \alpha_{0}^{l+m-n}\right)+n\left(\alpha_{1}^{m-n} \alpha_{1}^{l+m}-\alpha_{0}^{m-n} \alpha_{0}^{l+m}\right)\right) \\
& \quad \text { and } \quad-m^{2}(m-1)\left(\alpha_{1}^{l+m}-\alpha_{0}^{l+m}\right) .
\end{aligned}
$$

It follows that the normally ordered invariants do not commute with the generators of the Virasoro algebra, and that, again, the anomalies which arise are neither themselves invariant nor do they vanish on the physical subspace. Again, they are not simply multiples of the identity, and in fact, for invariants of higher tensor rank may even be polynomials in annihilation and creation operators of arbitrary rank. Hence, the question of whether 
canonical quantization encodes the geometric content of the Nambu-Goto string has to be answered in the negative.

Similar problems occur when the gauge is fixed to the light-cone gauge. One of the reasons for the necessity of a critical dimension in the canonical approach is that in this gauge, the generators of the Poincaré group only close as a Lie algebra (at least weakly) in $d=26$. Calculating the action of these generators on invariant charges we also find anomalies which, however, contrary to those arising in a commutator of two generators, do not vanish in some critical dimension. Hence, the canonically quantized invariant charges do no longer transform covariantly in this approach. For details see [1].

The results presented here show that the canonical approach and the algebraic quantization are inequivalent. This means in particular, that the usual Fock space does not yield a suitable representation of the algebra of invariant charges. Lately, a representation providing an alternative to the Fock space construction was proposed in [12].

To conclude, some comments on the relation between the algebra of the invariant charges and the so-called DDF operators [13] seem to be appropriate. Since the latter commute with all Virasoro generators (in the conformal gauge), they are sometimes considered to provide a "canonical algebra of invariant quantities". However, the crucial point is that the construction rules for a genuine invariant quantity must be gauge-independent. This requirement is met by the algebra of invariant charges: regardless of whether one starts from a conformal or from some other parametrization, the rules for the construction of the algebra of invariant charges are the same (and the charges are invariant under arbitrary reparametrizations). In contrast to this, the construction of the DDF operators relies on choosing the conformal gauge, and hence, by the above criterion, the DDF operators are not genuinely invariant ${ }^{4}$

\section{Acknowledegment}

I would like to thank Professor K. Pohlmeyer, who provided the idea for this investigation and supervised my thesis on the subject. His critical comments on this manuscript are also gratefully acknowledged.

\footnotetext{
${ }^{4}$ Note added: In the meantime, a construction of classical DDF-like operators independent of a particular gauge has been given and it was shown that the classical invariant charges can be written as functionals of these operators [14. One main difficulty in using these operators as a starting point for quantization is to show that Lorentz symmetry can be kept after quantization.
} 


\section{A The classical relations at $\ell=2$}

$$
\begin{aligned}
& J^{P}=4^{-}: \quad 0=\left\{T_{2}, S_{2}\right\}_{4} \\
& J^{P}=3^{+}: \quad 0=\left\{T_{2}, T_{2}\right\}_{3}+i\left\{S_{2}, S_{1}\right\}_{3}-i 16\left(J_{1}^{3}\right)_{3} \\
& 0=\left\{S_{2}, S_{2}\right\}_{3}-i 2\left\{S_{2}, S_{1}\right\}_{3}+i 8\left(J_{1} \cdot T_{2}\right)_{3}+i 48\left(J_{1}^{3}\right)_{3} \\
& J^{P}=3^{-}: \quad 0=\left\{T_{2}, S_{2}\right\}_{3}-i\left\{T_{2}, S_{1}\right\}_{3}-i 8\left(J_{1} \cdot S_{2}\right)_{3} \\
& J^{P}=2^{-}: \quad 0=\left\{T_{2}, S_{2}\right\}_{2}+\frac{i}{3} \sqrt{\frac{7}{2}}\left\{T_{2}, S_{1}\right\}_{2}+i \frac{4}{3} \sqrt{14}\left(J_{1} \cdot S_{2}\right)_{2} \\
& J^{P}=1^{+}: \quad 0=\left\{S_{2}, S_{2}\right\}_{1}+i \sqrt{\frac{2}{3}}\left\{S_{2}, S_{1}\right\}_{1}+\frac{1}{6} \sqrt{5}\left\{S_{1}, S_{1}\right\}_{1} \\
& +i 16 \sqrt{\frac{2}{3}}\left(J_{1} \cdot T_{2}\right)_{1}+i 32 \sqrt{\frac{2}{15}}\left(J_{1} \cdot\left(J_{1}^{2}\right)_{0}\right)_{1}
\end{aligned}
$$

action of the exceptional element $B_{0}^{(1)}$ :

$$
\begin{aligned}
J^{P}=2^{+}:\left\{B_{0}^{(1)}, T_{2}\right\}_{2}= & i \sqrt{6}\left\{S_{2}, S_{1}\right\}_{2} \\
J^{P}=2^{-}:\left\{B_{0}^{(1)}, S_{2}\right\}_{2}= & -i 2 \sqrt{\frac{2}{3}}\left\{T_{2}, S_{1}\right\}_{2}-i 4 \sqrt{\frac{2}{3}}\left(J_{1} \cdot S_{2}\right)_{2}+12\left(J_{1} \cdot S_{1}\right)_{2} \\
J^{P}=1^{-}:\left\{B_{0}^{(1)}, S_{1}\right\}_{1}= & -i 6 \sqrt{\frac{2}{5}}\left\{T_{2}, S_{2}\right\}_{1}+2 \sqrt{\frac{3}{5}}\left\{T_{2}, S_{1}\right\}_{1}-24 \sqrt{\frac{3}{5}}\left(J_{1} \cdot S_{2}\right)_{1} \\
& +i 12 \sqrt{2}\left(J_{1} \cdot S_{1}\right)_{1} .
\end{aligned}
$$

Here,

$$
\begin{aligned}
\left\{X_{j_{1}}, Y_{j_{2}}\right\}_{j, m} & =\underbrace{\sum_{m_{1}=-j_{1}}^{j_{1}} \sum_{m_{2}=-j_{2}}^{j_{2}}}_{m_{1}+m_{2}=m}\left\langle j, m \mid j_{1}, m_{1} ; j_{2}, m_{2}\right\rangle\left\{X_{j_{1}, m_{1}}, Y_{j_{2}, m_{2}}\right\}, \\
\left(X_{j_{1}} \cdot Y_{j_{2}}\right)_{j, m} & =\underbrace{\sum_{m_{1}=-j_{1}}^{j_{1}} \sum_{m_{2}=-j_{2}}^{j_{2}}}_{m_{1}+m_{2}=m}\left\langle j, m \mid j_{1}, m_{1} ; j_{2}, m_{2}\right\rangle X_{j_{1}, m_{1}} \cdot Y_{j_{2}, m_{2}} .
\end{aligned}
$$




\section{B Normally ordered algebra elements}

$$
\begin{aligned}
& : \mathcal{Z}_{0 i j}^{(2)}: \quad=\frac{\mathfrak{m}^{3}}{2 \pi} \frac{1}{i} \sum_{n>0} \frac{1}{n}\left(\alpha_{i}^{-n} \alpha_{j}^{n}-\alpha_{j}^{-n} \alpha_{i}^{n}\right)
\end{aligned}
$$

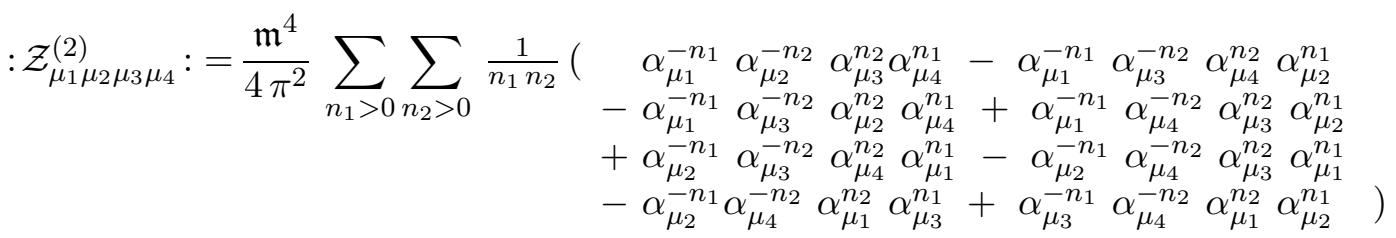

$$
\begin{aligned}
& -\frac{\mathfrak{m}^{4}}{4 \pi^{2}} \delta_{\mu_{1}, 0} \sum_{n_{1}>0} \sum_{n_{2}>0} \frac{1}{n_{1}\left(n_{1}+n_{2}\right)}\left(\begin{array}{c}
\alpha_{\mu_{2}}^{-n_{1}} \alpha_{\mu_{3}}^{-n_{2}} \alpha_{\mu_{4}}^{n_{1}+n_{2}}+\alpha_{\mu_{4}}^{-n_{1}} \alpha_{\mu_{3}}^{-n_{2}} \alpha_{\mu_{2}}^{n_{1}+n_{2}} \\
+\alpha_{\mu_{2}}^{-n_{1}-n_{2}} \alpha_{\mu_{4}}^{n_{1}} \alpha_{\mu_{3}}^{n_{2}}+\alpha_{\mu_{4}}^{-n_{1}-n_{2}} \alpha_{\mu_{2}}^{n_{1}} \alpha_{\mu_{3}}^{n_{2}}
\end{array}\right) \\
& -\frac{\mathfrak{m}^{4}}{4 \pi^{2}} \delta_{\mu_{2}, 0} \sum_{n_{1}>0} \sum_{n_{2}>0} \frac{1}{n_{1}\left(n_{1}+n_{2}\right)}\left(\begin{array}{c}
\alpha_{\mu_{3}}^{-n_{1}} \alpha_{\mu_{4}}^{-n_{2}} \alpha_{\mu_{1}}^{n_{1}+n_{2}}+\alpha_{\mu_{1}}^{-n_{1}} \alpha_{\mu_{4}}^{-n_{2}} \alpha_{\mu_{3}}^{n_{1}+n_{2}} \\
+\alpha_{\mu_{3}}^{-n_{1}-n_{2}} \alpha_{\mu_{1}}^{n_{1}} \alpha_{\mu_{4}}^{n_{2}}+\alpha_{\mu_{1}}^{-n_{1}-n_{2}} \alpha_{\mu_{3}}^{n_{1}} \alpha_{\mu_{4}}^{n_{2}}
\end{array}\right) \\
& -\frac{\mathfrak{m}^{4}}{4 \pi^{2}} \delta_{\mu_{3}, 0} \sum_{n_{1}>0} \sum_{n_{2}>0} \frac{1}{n_{1}\left(n_{1}+n_{2}\right)}\left(\begin{array}{c}
\alpha_{\mu_{4}}^{-n_{1}} \alpha_{\mu_{1}}^{-n_{2}} \alpha_{\mu_{2}}^{n_{1}+n_{2}}+\alpha_{\mu_{2}}^{-n_{1}} \alpha_{\mu_{1}}^{-n_{2}} \alpha_{\mu_{4}}^{n_{1}+n_{2}} \\
+\alpha_{\mu_{2}}^{-n_{1}-n_{2}} \alpha_{\mu_{4}}^{n_{1}} \alpha_{\mu_{1}}^{n_{2}}+\alpha_{\mu_{4}}^{-n_{1}-n_{2}} \alpha_{\mu_{2}}^{n_{1}} \alpha_{\mu_{1}}^{n_{2}}
\end{array}\right) \\
& -\frac{\mathfrak{m}^{4}}{4 \pi^{2}} \delta_{\mu_{4}, 0} \sum_{n_{1}>0} \sum_{n_{2}>0} \frac{1}{n_{1}\left(n_{1}+n_{2}\right)}\left(\begin{array}{c}
\alpha_{\mu_{1}}^{-n_{1}} \alpha_{\mu_{2}}^{-n_{2}} \alpha_{\mu_{3}}^{n_{1}+n_{2}}+\alpha_{\mu_{3}}^{-n_{1}} \alpha_{\mu_{2}}^{-n_{2}} \alpha_{\mu_{1}}^{n_{1}+n_{2}} \\
+\alpha_{\mu_{1}}^{-n_{1}-n_{2}} \alpha_{\mu_{2}}^{n_{1}} \alpha_{\mu_{3}}^{n_{2}}+\alpha_{\mu_{3}}^{-n_{1}-n_{2}} \alpha_{\mu_{2}}^{n_{1}} \alpha_{\mu_{1}}^{n_{2}}
\end{array}\right) \\
& +\frac{\mathfrak{m}^{4}}{4 \pi^{2}} \delta_{\mu_{1}, 0} \sum_{n_{1}>0} \sum_{n_{2}>0} \frac{1}{n_{1} n_{2}}\left(\alpha_{\mu_{2}}^{-n_{1}} \alpha_{\mu_{4}}^{-n_{2}} \alpha_{\mu_{3}}^{n_{1}+n_{2}}+\alpha_{\mu_{3}}^{-n_{1}-n_{2}} \alpha_{\mu_{2}}^{n_{1}} \alpha_{\mu_{4}}^{n_{2}}\right) \\
& +\frac{\mathfrak{m}^{4}}{4 \pi^{2}} \delta_{\mu_{2}, 0} \sum_{n_{1}>0} \sum_{n_{2}>0} \frac{1}{n_{1} n_{2}}\left(\alpha_{\mu_{1}}^{-n_{1}} \alpha_{\mu_{3}}^{-n_{2}} \alpha_{\mu_{4}}^{n_{1}+n_{2}}+\alpha_{\mu_{4}}^{-n_{1}-n_{2}} \alpha_{\mu_{1}}^{n_{1}} \alpha_{\mu_{3}}^{n_{2}}\right) \\
& +\frac{\mathfrak{m}^{4}}{4 \pi^{2}} \delta_{\mu_{3}, 0} \sum_{n_{1}>0} \sum_{n_{2}>0} \frac{1}{n_{1} n_{2}}\left(\alpha_{\mu_{2}}^{-n_{1}} \alpha_{\mu_{4}}^{-n_{2}} \alpha_{\mu_{1}}^{n_{1}+n_{2}}+\alpha_{\mu_{1}}^{-n_{1}-n_{2}} \alpha_{\mu_{2}}^{n_{1}} \alpha_{\mu_{4}}^{n_{2}}\right) \\
& +\frac{\mathfrak{m}^{4}}{4 \pi^{2}} \delta_{\mu_{4}, 0} \sum_{n_{1}>0} \sum_{n_{2}>0} \frac{1}{n_{1} n_{2}}\left(\alpha_{\mu_{1}}^{-n_{1}} \alpha_{\mu_{3}}^{-n_{2}} \alpha_{\mu_{2}}^{n_{1}+n_{2}}+\alpha_{\mu_{2}}^{-n_{1}-n_{2}} \alpha_{\mu_{1}}^{n_{1}} \alpha_{\mu_{3}}^{n_{2}}\right) \\
& +\frac{\mathfrak{m}^{4}}{4 \pi^{2}} \delta_{\mu_{1}, 0} \delta_{\mu_{2}, 0} \sum_{n_{1}>0} \frac{1}{n_{1}^{2}}\left(\alpha_{\mu_{3}}^{-n_{1}} \alpha_{\mu_{4}}^{n_{1}}+\alpha_{\mu_{4}}^{-n_{1}} \alpha_{\mu_{3}}^{n_{1}}\right) \\
& -2 \frac{\mathfrak{m}^{4}}{4 \pi^{2}} \delta_{\mu_{1}, 0} \delta_{\mu_{3}, 0} \sum_{n_{1}>0} \frac{1}{n_{1}^{2}}\left(\alpha_{\mu_{2}}^{-n_{1}} \alpha_{\mu_{4}}^{n_{1}}+\alpha_{\mu_{4}}^{-n_{1}} \alpha_{\mu_{2}}^{n_{1}}\right) \\
& +\frac{\mathfrak{m}^{4}}{4 \pi^{2}} \delta_{\mu_{1}, 0} \delta_{\mu_{4}, 0} \sum_{n_{1}>0} \frac{1}{n_{1}^{2}}\left(\alpha_{\mu_{2}}^{-n_{1}} \alpha_{\mu_{3}}^{n_{1}}+\alpha_{\mu_{3}}^{-n_{1}} \alpha_{\mu_{2}}^{n_{1}}\right) \\
& +\frac{\mathfrak{m}^{4}}{4 \pi^{2}} \delta_{\mu_{2}, 0} \delta_{\mu_{3}, 0} \sum_{n_{1}>0} \frac{1}{n_{1}^{2}}\left(\alpha_{\mu_{1}}^{-n_{1}} \alpha_{\mu_{4}}^{n_{1}}+\alpha_{\mu_{4}}^{-n_{1}} \alpha_{\mu_{1}}^{n_{1}}\right) \\
& -2 \frac{\mathfrak{m}^{4}}{4 \pi^{2}} \delta_{\mu_{2}, 0} \delta_{\mu_{4}, 0} \sum_{n_{1}>0} \frac{1}{n_{1}^{2}}\left(\alpha_{\mu_{1}}^{-n_{1}} \alpha_{\mu_{3}}^{n_{1}}+\alpha_{\mu_{3}}^{-n_{1}} \alpha_{\mu_{1}}^{n_{1}}\right) \\
& +\frac{\mathfrak{m}^{4}}{4 \pi^{2}} \delta_{\mu_{3}, 0} \delta_{\mu_{4}, 0} \sum_{n_{1}>0} \frac{1}{n_{1}^{2}}\left(\alpha_{\mu_{1}}^{-n_{1}} \alpha_{\mu_{2}}^{n_{1}}+\alpha_{\mu_{2}}^{-n_{1}} \alpha_{\mu_{1}}^{n_{1}}\right)
\end{aligned}
$$




\section{The anomalies for the relations involving $B_{0}^{(1)}$}

$$
\begin{aligned}
& \begin{array}{c|c}
J^{P} & \text { anomalies with } m=-J \\
\hline 2^{+} & \frac{\hbar}{2 \pi \alpha^{\prime}} \frac{\mathfrak{m}^{4}}{(2 \pi)^{2}} \sum_{n, m>0} \frac{4}{n m}\left(2 \alpha_{-}^{-n} \alpha_{+}^{-m} \alpha_{-}^{m} \alpha_{-}^{n}-2 \alpha_{-}^{-n} \alpha_{-}^{-m} \alpha_{+}^{m} \alpha_{-}^{n}\right. \\
& \left.+\alpha_{3}^{-n} \alpha_{3}^{-m} \alpha_{-}^{m} \alpha_{-}^{n}-\alpha_{-}^{-n} \alpha_{-}^{-m} \alpha_{3}^{m} \alpha_{3}^{n}\right)
\end{array} \\
& 2^{-} \quad \frac{\hbar}{2 \pi \alpha^{\prime}} \frac{\mathfrak{m}^{4}}{(2 \pi)^{2}} \sum_{n, m>0} \frac{4 i}{n m}\left(2 \alpha_{-}^{-n} \alpha_{3}^{-m} \alpha_{0}^{m} \alpha_{-}^{n}-2 \alpha_{-}^{-n} \alpha_{0}^{-m} \alpha_{3}^{m} \alpha_{-}^{n}\right. \\
& +\alpha_{0}^{-n} \alpha_{-}^{-m} \alpha_{3}^{m} \alpha_{-}^{n}-\alpha_{-}^{-n} \alpha_{-}^{-m} \alpha_{3}^{m} \alpha_{0}^{n} \\
& \left.+\alpha_{0}^{-n} \alpha_{3}^{-m} \alpha_{-}^{m} \alpha_{-}^{n}-\alpha_{-}^{-n} \alpha_{3}^{-m} \alpha_{-}^{m} \alpha_{0}^{n}\right) \\
& +\frac{\hbar}{2 \pi \alpha^{\prime}} \frac{\mathfrak{m}^{4}}{(2 \pi)^{2}} \sum_{n, m>0} \frac{4 i}{n(n+m)}\left(-2 \alpha_{3}^{-n} \alpha_{-}^{-m} \alpha_{-}^{n+m}+2 \alpha_{-}^{-n-m} \alpha_{3}^{n} \alpha_{-}^{m}\right. \\
& +\alpha_{-}^{-n} \alpha_{-}^{-m} \alpha_{3}^{n+m}-\alpha_{3}^{-n-m} \alpha_{-}^{n} \alpha_{-}^{m} \\
& \left.+\alpha_{-}^{-n} \alpha_{3}^{-m} \alpha_{-}^{n+m}-\alpha_{-}^{-n-m} \alpha_{-}^{n} \alpha_{3}^{m}\right) \\
& 1^{-} \quad \frac{\hbar}{2 \pi \alpha^{\prime}} \frac{\mathfrak{m}^{4}}{(2 \pi)^{2}} \sum_{n, m>0} \frac{4}{n m} \frac{3}{5}\left(6 \alpha_{0}^{-n} \alpha_{-}^{-m} \alpha_{-}^{m} \alpha_{+}^{n}-6 \alpha_{+}^{-n} \alpha_{-}^{-m} \alpha_{-}^{m} \alpha_{0}^{n}\right. \\
& +3 \alpha_{0}^{-n} \alpha_{-}^{-m} \alpha_{3}^{m} \alpha_{3}^{n}-3 \alpha_{3}^{-n} \alpha_{-}^{-m} \alpha_{3}^{m} \alpha_{0}^{n} \\
& +3 \alpha_{0}^{-n} \alpha_{3}^{-m} \alpha_{-}^{m} \alpha_{3}^{n}-3 \alpha_{3}^{-n} \alpha_{3}^{-m} \alpha_{-}^{m} \alpha_{0}^{n} \\
& -2 \alpha_{0}^{-n} \alpha_{3}^{-m} \alpha_{3}^{m} \alpha_{-}^{n}+2 \alpha_{-}^{-n} \alpha_{3}^{-m} \alpha_{3}^{m} \alpha_{0}^{n} \\
& +\alpha_{0}^{-n} \alpha_{-}^{-m} \alpha_{+}^{m} \alpha_{-}^{n}-\alpha_{-}^{-n} \alpha_{-}^{-m} \alpha_{+}^{m} \alpha_{0}^{n} \\
& \left.+\alpha_{0}^{-n} \alpha_{+}^{-m} \alpha_{-}^{m} \alpha_{-}^{n}-\alpha_{-}^{-n} \alpha_{+}^{-m} \alpha_{-}^{m} \alpha_{0}^{n}\right) \\
& +\frac{\hbar}{2 \pi \alpha^{\prime}} \frac{\mathfrak{m}^{4}}{(2 \pi)^{2}} \sum_{n, m>0} \frac{4}{n(n+m)} \frac{3}{5}\left(6 \alpha_{+}^{-n} \alpha_{-}^{-m} \alpha_{-}^{n+m}-6 \alpha_{-}^{-n-m} \alpha_{+}^{n} \alpha_{-}^{m}\right. \\
& +3 \alpha_{3}^{-n} \alpha_{3}^{-m} \alpha_{-}^{n+m}-3 \alpha_{-}^{-n-m} \alpha_{3}^{n} \alpha_{3}^{m} \\
& +3 \alpha_{3}^{-n} \alpha_{-}^{-m} \alpha_{3}^{n+m}-3 \alpha_{3}^{-n-m} \alpha_{3}^{n} \alpha_{-}^{m} \\
& -2 \alpha_{-}^{-n} \alpha_{3}^{-m} \alpha_{3}^{n+m}+2 \alpha_{3}^{-n-m} \alpha_{-}^{n} \alpha_{3}^{m} \\
& +\alpha_{-}^{-n} \alpha_{+}^{-m} \alpha_{-}^{n+m}-\alpha_{-}^{-n-m} \alpha_{-}^{n} \alpha_{+}^{m} \\
& \left.+\alpha_{-}^{-n} \alpha_{-}^{-m} \alpha_{+}^{n+m}-\alpha_{+}^{-n-m} \alpha_{-}^{n} \alpha_{-}^{m}\right)
\end{aligned}
$$




\section{References}

[1] D. Bahns, Die Invariantenalgebra des Nambu-Goto-Strings in Erzeugungs- und Vernichtungsoperatoren, Diplomarbeit (in German), Universität Freiburg (1999).

[2] K. Pohlmeyer, "A Group-theoretical Approach to the Quantization of the Free Relativistic Closed String", Physics Letters 119 B (1982).

[3] K.-H. Rehren, Zur invarianten Quantisierung des relativistischen freien Strings, Doctoral thesis (in German), Universität Freiburg (1984).

[4] K. Pohlmeyer, K.-H. Rehren, "Algebraic Properties of the Invariant Charges of the Nambu-Goto-Theory", Commun. Math. Phys. 105 (1986).

[5] K. Pohlmeyer, K.-H. Rehren, "The Invariant Charges of the Nambu-Goto Theory: Their Geometric Origin and Their Completeness", Commun. Math. Phys. 114 (1988).

[6] K. Pohlmeyer, "The Nambu-Goto-Theory of Closed Bosonic Strings Moving in $1+$ 3-Dimensional Minkowski Space: The Quantum Algebra of Observables", Annalen Phys. 8 (1999) arXiv:hep-th/9805057.

[7] K. Pohlmeyer, M. Trunk, "The Invariant Charges of the Nambu-Goto String Theory: Quantization of Non-Additive Composition Laws", Int.J.Mod.Phys. A19 (2004) arXiv:hep-th/0206061.

[8] G. Handrich, C. Nowak, The Nambu-Goto-Theory of Closed Bosonic Strings Moving in 1+3-Dimensional Minkowski Space: The Construction of the Quantum Algebra of Observables Up to Degree Five, Annalen Phys. 8 (1999) arxiv:hep-th/9807231.

[9] C. Meusburger, Die Quantisierung der Invariantenalgebra des Nambu-Goto-Strings mit Hilfe einer konkreten Realisierung, Diplomarbeit (in German), Universität Freiburg (2001)

[10] C. Meusburger, K.-H. Rehren, "Algebraic quantization of the closed bosonic string", Commun. Math. Phys. 237 (2003) arXiv:math-ph/0202041.

[11] T. Kornhaß, Klassische Erhaltungsgrößen auf verzweigten Stringtrajektorien der Nambu-Goto-Theorie, Doctoral thesis (in German), Universität Freiburg (1991).

[12] T. Thiemann, "The LQG - String: Loop Quantum Gravity Quantization of String Theory I. Flat Target Space" arXiv:hep-th/0401172.

[13] E. Del Giudice, P. Di Vecchia and S. Fubini, "General Properties Of The Dual Resonance Model", Annals Phys. 70 (1972).

[14] U. Schreiber, "DDF and Pohlmeyer invariants of (super)string" arXiv:hep-th/0403260. 